TRANSACTIONS OF THE

AMERICAN MATHEMATICAL SOCIETY

Volume 353, Number 6, Pages 2531-2556

S 0002-9947(01)02779-9

Article electronically published on February 15, 2001

\title{
COMPLEXIFICATIONS OF SYMMETRIC SPACES AND JORDAN THEORY
}

\author{
WOLFGANG BERTRAM
}

\begin{abstract}
Generalizing Hermitian and pseudo-Hermitian spaces, we define twisted complex symmetric spaces, and we show that they correspond to an algebraic object called Hermitian Jordan triple products. The main topic of this work is to investigate the class of real forms of twisted complex symmetric spaces, called the category of symmetric spaces with twist. We show that this category is equivalent to the category of all real Jordan triple systems, and we can use a work of B.O. Makarevič in order to classify the irreducible spaces. The classification shows that most irreducible symmetric spaces have exactly one twisted complexification. This leads to open problems concerning the relation of Jordan and Lie triple systems.
\end{abstract}

\section{INTRODUCTION}

0.1. Straight and twisted complexifications. The Euclidean vector space $\left(\mathbb{R}^{n}, \sum_{i} x_{i} y_{i}\right)$ can be "complexified" in two ways: first, we have the extension $\left(\mathbb{C}^{n}, \sum_{i} z_{i} w_{i}\right)$ of the scalar product to a complex bilinear form on $\mathbb{C}^{n}$, and second, we have the "Hermitification" $\left(\mathbb{C}^{n}, \sum_{i} z_{i} \bar{w}_{i}\right)$ which yields a scalar product on $\mathbb{C}^{n}$. Of course, this works for any bilinear form $b$ on $\mathbb{R}^{n}$; let us call $b_{\mathbb{C}}(z, w)$ its "straight" (complex bilinear) and $b_{\mathbb{C}}(z, \bar{w})$ its "twisted" complexification.

In this paper we investigate an analogue of this construction in a situation which geometrically is much less trivial due to the presence of curvature. A natural class of spaces to look at here is the class of symmetric spaces since these are characterized by one fundamental invariant, namely by the curvature tensor $R$ itself which satisfies the algebraic identities of a Lie triple system (LTS; cf. Def. 1.1.1). It is almost trivial that any such space admits (locally) a straight complexification given by the $\mathbb{C}$-trilinear extension $R_{\mathbb{C}}$ of $R$; from a group theoretic point of view this can be interpreted as the complexification of the homogeneous symmetric space $M=G / H$ locally by the complex symmetric space $M_{\mathbb{C}}=G_{\mathbb{C}} / H_{\mathbb{C}}$, where $G_{\mathbb{C}}$ and $H_{\mathbb{C}}$ are suitable complexifications of the Lie groups $G$ and $H$.

The definition of a Hermitification or twisted complexification of a symmetric space $M$ is less obvious. In the Riemannian case it should amount to realize $M$ as a real form of a Hermitian symmetric space. For the correct definition in the general (non-Riemannian or even possibly nilpotent or solvable) case, recall (cf. [Hel62] or [L669) that for any (pseudo-)Hermitian symmetric space we have the relation

$$
R(\mathcal{J} X, Y) Z=-R(X, \mathcal{J} Y) Z
$$

Received by the editors February 19, 1998.

2000 Mathematics Subject Classification. Primary 17C36, 53C15; Secondary 22E15, 53B35.

Key words and phrases. Symmetric space, complexification, Jordan and Lie triple system. 
for the curvature $R$, the invariant almost complex structure $\mathcal{J}$ and vector fields $X, Y, Z$. We consider this relation as fundamental and take it as the defining relation of a twisted complex symmetric space. A twisted complexification is then a (local) imbedding of $M$ as a real form of a twisted complex symmetric space. For instance, the unit disc $\mathrm{SU}(1,1) / \mathrm{SO}(2)$ is a twisted complexification of its real form $M=]-1,1[\cong \mathbb{R}$; the complex projective space $\mathrm{U}(n+1) /(\mathrm{U}(n) \times \mathrm{U}(1))$ is a twisted complexification of the real projective space $M=\mathrm{O}(n+1) /(\mathrm{O}(n) \times \mathrm{O}(1))$; the variety $\operatorname{Gl}(2 n, \mathbb{R}) / \mathrm{Gl}(n, \mathbb{C})$ of complex structures on $\mathbb{R}^{2 n}$ is a twisted complexification of the symmetric space of group type $M=\mathrm{Gl}(n, \mathbb{R})$ (cf. Ex. 1.3.7).

We do not claim that any symmetric space admits a twisted complexification and, if it does, that it is unique. In fact, there is a very subtle curvature obstruction conveniently described in terms of Jordan theory: it would be tempting to define the twisted complexification of a LTS $R$ by $R_{\mathbb{C}}(X, \bar{Y}) Z$, but this does not work. However, we will see that in the Jordan category this idea works perfectly well.

0.2. The Jordan theoretic viewpoint. In the late sixties Max Koecher found a relation between Hermitian symmetric spaces and certain Jordan algebras; this was put into a more conceptual framework by O. Loos in [Lo77] where a bijection between bounded symmetric domains and the so-called positive Hermitian Jordan triple systems is established (cf. also [Sa80]). We generalize this correspondence in a first step to twisted complex symmetric spaces: they are equivalent to Hermitian Jordan triple systems (Th. 1.3.6), and in a second step to their real forms: they are equivalent to general real Jordan triple systems (Th. 2.2.6). The idea is simple. We introduce the structure tensor of a twisted complex symmetric space with curvature tensor $R$ and almost complex structure $\mathcal{J}$ by the formula

$$
T(X, Y, Z):=\frac{1}{2}(R(X, Y) Z+\mathcal{J} R(X, \mathcal{J} Y) Z)
$$

and prove that it is a Hermitian Jordan triple system (Prop. 1.3.2). It can be restricted to real forms (Lemma 2.2.1) and it contains all information in order to reconstruct (locally) the twisted complex symmetric space from its real form (Th. 2.2.2). This is due to the remarkable relation

$$
T(X, Y, Z)-T(Y, X, Z)=R(X, Y) Z ;
$$

we say that $T$ is a Jordan extension of the curvature $R$. We are thus lead to the category of symmetric spaces with a Jordan extension, called the category of symmetric spaces with twist (Def. 2.2.5); by our result (Th. 2.2.6) this category is equivalant to the category of symmetric spaces with a (local) twisted complexification.

0.3. Twisted para-complexifications. Our method can also be applied to paracomplexifications; these are defined in the same way as complexifications, but using a structure with $J^{2}=$ id instead of $J^{2}=-$ id. They lead to a class of symmetric spaces generalizing the para-Hermitian symmetric spaces investigated by Kozai and Kaneyuki (cf. KanKo85]). It turns out that a symmetric space admits a twisted para-complexification if and only if it admits a twisted complexification (Th. 2.2.6). For instance, the one-sheeted hyperboloid is the twisted para-complexification of $\mathbb{R}$ corresponding to the twisted complexification given by the unit disc.

0.4. Classification. First, we classify invariant complex and para-complex structures on simple symmetric spaces; they are all either straight or twisted, and we obtain a partition of simple symmetric spaces into five classes (Th. 3.1). The result 
is essentially known by work of S. Koh [Koh65, but our proof is much shorter and more conceptual.

Next we come back to the problem mentioned above (end of Section 0.1): given a symmetric space $M=G / H$, how many twisted (para-)complexifications are there, if any, and how are they described as homogeneous spaces? In Chapter 4 we present the classification of simple twisted (para-)complexifications of symmetric spaces of classical type. The result is rather surprising:

A. (Existence) Every (modulo possibly a central extension) irreducible symmetric space of classical type admits a twisted complexification. (The central extension is needed e.g. in the group case $M=\mathrm{Sl}(n, \mathbb{R})$ which does not admit a twisted complexification, but its central extension $\operatorname{Gl}(n, \mathbb{R})$ does; see above.)

B. (Unicity) The number of inequivalent twisted complexifications in the situation of Th. A is either 1, 2 or 3, and "generically" it is equal to 1.

On an algebraic level, Observations A and B are equivalent to the statement that the algebraic Jordan-Lie functor assigning to a Jordan triple system $T$ the Lie triple system

$$
R_{T}(X, Y) Z:=T(X, Y, Z)-T(Y, X, Z)
$$

is (for the classical types) "generically bijective". This fact has already been discussed by E. Neher ([N85]) by using both the classification of Lie triple systems (B57]) and the classification of Jordan triple systems ([N80], [N81]), where the exceptional spaces are also considered. A classification-free approach to the JordanLie functor in the compact case is due to O. Loos ( $[$ Lo85] $)$. Our method of classification is different from the one used by E. Neher; it follows closely the classification of open symmetric orbits in symmetric $R$-spaces by B.O. Makarevič ([Ma73]).

0.5. Integrability. It is well-known that invariant almost complex structures on symmetric spaces are integrable (cf. KoNo69, Prop. X.6.5]); thus the twisted complex symmetric spaces are actually complex manifolds and the real forms considered here are their real forms as manifolds. However, the theory becomes clearer if in a first step one does not use this fact. We use it in a second step ([Be0] $)$ where we introduce the conformal group of a symmetric space with twist and characterize it by a theorem generalizing at the same time the fundamental theorem of projective geometry and the Liouville theorem for Jordan algebras (Be96a Th. 2.3.1]). Finally, we have in mind, applications of the structure theory developed here and in [Be98], [Be00] to harmonic analysis on symmetric spaces with twist.

Acknowledgement. I thank Joachim Hilgert for many helpful discussions.

\section{The StruCture tensor of A TWISTED COMPleX SYMMETRIC SPACE}

1.1. The Lie functor for symmetric spaces. A symmetric space (with base point) is a homogeneous space $M=G / H$ where $G$ is a Lie group and $H$ an open subgroup of the group of fixed points of some non-trivial involution $\sigma$ of $G$. The base point $e H \in M$ is denoted by $o$.

Morphisms of symmetric spaces (with base point) are smooth maps $\varphi: M \rightarrow$ $M^{\prime}$ of symmetric spaces which are base-point preserving and compatible with the canonical connections $\nabla$ and $\nabla^{\prime}$ associated to $M$ and $M^{\prime}$ (cf. [Lo69]).

The infinitesimal object associated to a symmetric space $M=G / H$ is the -1eigenspace $\mathfrak{q}$ in the eigenspace-decomposition $\mathfrak{g}=\mathfrak{h} \oplus \mathfrak{q}$ of the Lie algebra $\mathfrak{g}$ of 
$G$ under the differential $\dot{\sigma}$ of $\sigma$ at the origin. If $X, Y, Z \in \mathfrak{q}$, then $[X, Y, Z]:=$ $R(X, Y) Z:=[[X, Y], Z]$ again belongs to $\mathfrak{q}$, and we have the rules

(LT1): $R(X, Y)=-R(Y, X)$

(LT2): $[X, Y, Z]+[Y, Z, X]+[Z, X, Y]=0$,

(LT3): $R(U, V)[X, Y, Z]=[R(U, V) X, Y, Z]+[X, R(U, V) Y, Z]+[X, Y, R(U, V) Z]$.

Condition (LT3) means that $R(U, V)$ is a derivation of the triple bracket. If we identify $\mathfrak{q}$ with the tangent space $T_{o} M$ via the bijective evaluation map $e v_{o}: \mathfrak{q} \rightarrow$ $T_{o} M, X \mapsto X_{o}$, the triple product $[X, Y, Z]$ of $\mathfrak{q}$ is (up to a sign) the curvature tensor $R$ of the canonical connection of $M$, evaluated at $o$ ([Lo69] II.2.5]; we use a different sign in order to simplify formulas).

Definition 1.1.1. A vector space together with a trilinear map $\mathfrak{q} \times \mathfrak{q} \times \mathfrak{q} \rightarrow \mathfrak{q}$, $(X, Y, Z) \mapsto[X, Y, Z]=R(X, Y) Z$ satisfying (LT1), (LT2) and (LT3) is called a Lie triple system (abbreviated LTS). Morphisms of Lie triple systems are linear maps which are compatible with the respective triple products.

It is a matter of straightforward verification (cf. [Lo69, Prop. II.2.3]) that for any LTS $\mathfrak{q}$, the direct sum $\mathfrak{g}$ of $\mathfrak{q}$ and the algebra $\operatorname{Der}(\mathfrak{q})$ of derivations of $\mathfrak{q}$ is a Lie algebra with the Lie bracket defined in the obvious way, such that the abstract LTS $\mathfrak{q}$ is imbedded as the sub-triple system $\mathfrak{q}$ of $\mathfrak{g}$ with its ordinary triple Lie bracket $[X, Y, Z]=[[X, Y], Z]$. The subalgebra $[\mathfrak{q}, \mathfrak{q}] \oplus \mathfrak{q} \subset \mathfrak{g}$ is called the standard-imbedding of the LTS q.

Theorem 1.1.2. The functor of evaluating the curvature tensor at the base point defines an equivalence of the categories of connected simply connected symmetric spaces with base point and the category of finite-dimensional real Lie triple systems.

Proof [Lo69, Th. II.4.12].

We will only need a weaker version of Theorem 1.1.2. Two symmetric spaces $M$, $M^{\prime}$ with base points $o, o^{\prime}$ are called locally isomorphic if there exist neighbourhoods $U, U^{\prime}$ of the base points and an affine diffeomorphism $\varphi: U \rightarrow U^{\prime}$ with $\varphi(o)=o^{\prime}$. This is an equivalence relation, and we may speak of local equivalence classes or germs of symmetric spaces and of their morphisms.

Theorem 1.1.3. The category of germs of symmetric spaces is equivalent to the category of finite-dimensional real Lie triple systems.

Proof. We define the functor from Lie triple systems to germs of symmetric spaces by taking the local equivalence class of the connected simply connected space given by Theorem 1.1.2. Then the claim follows from this theorem.

If $G$ and $H$ are complex Lie groups and $\sigma$ is a holomorphic involution of $G$, then $M$ is a complex symmetric space, $\mathfrak{q}$ is a complex vector space and the associated Lie triple-product is a $\mathbb{C}$-trilinear map. A complex version of Theorem 1.1.3. holds.

The main point in the proof of Theorem 1.1.2 is to show that every morphism of Lie triple systems extends to a morphism of the corresponding symmetric spaces. Here, it is important to use the correct notion of morphisms of symmetric spaces; the notion of (weakly) equivariant maps of symmetric spaces (cf. [Sa80 p. 47]) is in general not the correct one, which is the cause of some problems in the presentation given in Sa80. The relation between morphisms of symmetric spaces and weakly equivariant maps (on the algebraic level) is dicussed in Jac51. 
1.2. Straight and twisted almost complex structures. An almost complex structure on a manifold $M$ is a tensor field $\mathcal{J}$ of type $(1,1)$ (i.e., $\mathcal{J}=\left(\mathcal{J}_{p}\right)_{p \in M}$ where $\mathcal{J}_{p}$ is an endomorphism of the tangent space $\left.T_{p} M\right)$ such that $\mathcal{J}_{p}^{2}=-\mathrm{id}_{T_{p} M}$ for all $p \in M$.

If $M=G / H$ is a symmetric space, we say that $\mathcal{J}$ is invariant if it is invariant under the action of the group $G$. Then $J:=\mathcal{J}_{o}$ is $H$-invariant in the sense that $J$ commutes with all tangent maps $T_{o} h, h \in H$, and any $J$ having this property can be extended uniquely to a $G$-invariant tensor field. Thus $G$-invariant almost complex structures correspond to $H$-invariant complex structures on $T_{o} M$. If we identify $\mathfrak{q}$ with $T_{o} M$ as above, the first isotropy representation of the Lie algebra $\mathfrak{h}$ of $H$ is just the usual action of $\mathfrak{h}$ on $\mathfrak{q}$ by the adjoint representation. If $J$ is $H$-invariant on $\mathfrak{q}=T_{o} M$, then it is $\mathfrak{h}$-invariant:

$$
\forall D \in \mathfrak{h}: \quad D \circ J=J \circ D .
$$

Since $[\mathfrak{q}, \mathfrak{q}] \subset \mathfrak{h},(I)$ implies the weaker condition

$$
\forall X, Y, Z \in \mathfrak{q}: \quad[X, Y, J Z]=J[X, Y, Z] .
$$

If $\left(\mathrm{I}^{\prime}\right)$ holds, we say that $J$ is an invariant complex structure on the LTS $\mathfrak{q}$. Lie triple systems with invariant complex structure form a category whose morphisms are $\mathbb{C}$-linear LTS-homomorphisms.

Proposition 1.2.1. The category of LTS with invariant complex structure is equivalent to the category of germs of symmetric spaces with invariant almost complex structure, morphisms being morphisms of symmetric spaces which are almostholomorphic, i.e. compatible with the respective almost complex structures.

Proof. It is clear that the objects of both categories correspond to each other under the bijection from Theorem 1.2.3. The differential at the base point of an almost-holomorphic morphism of symmetric spaces is clearly $\mathbb{C}$-linear and is thus a morphism of twisted complex LTS. Let us show that conversely a $\mathbb{C}$-linear LTShomomorphism $\dot{\varphi}$ induces an almost holomorphic morphism $\varphi$ of germs of symmetric spaces. In fact, the differential of $\varphi$ at the point $o$ is $\mathbb{C}$-linear by assumption, and from the condition $\varphi \circ \exp (X)=\exp (\dot{\varphi} X) \circ \varphi$ for $X \in \mathfrak{q}$ ([Lo69, Th. II.4.6]) it follows that the differential of $\varphi$ at the point $p=\exp (X) \cdot o$ is $\mathbb{C}$-linear.

Definition 1.2.2. An invariant complex structure $J$ on a LTS $\mathfrak{q}$ is called straight if

$$
\forall X, Y, Z \in \mathfrak{q}: \quad[J X, Y, Z]=[X, J Y, Z]
$$

holds, and twisted if

$$
\forall X, Y, Z \in \mathfrak{q}: \quad[J X, Y, Z]=-[X, J Y, Z]
$$

holds. A straight complex symmetric space $M=G / H$ is a symmetric space with an invariant almost complex structure $\mathcal{J}$ such that $R(\mathcal{J} X, Y) Z=R(X, \mathcal{J} Y) Z$, and a twisted complex symmetric space is a symmetric space with an invariant almost complex structure such that $R(\mathcal{J} X, Y) Z=-R(X, \mathcal{J} Y) Z$, where $R$ is the curvature tensor and $X, Y, Z$ are vector fields on $M$.

Proposition 1.2.3. An invariant complex structure $J$ on a LTS $\mathfrak{q}$ is straight if and only if the triple product is $J$-linear in all three variables. 
Proof. We only have to show that a straight invariant complex structure is $J$ trilinear. By invariance, it is $J$-linear in the last variable. Using the the Jacobiidentity, (S) and (I') yield

$$
0=[J X, Y, Z]+[J Z, X, Y]+J[Y, Z, X] .
$$

We now add up the three equations obtained by cyclic permutation of $X, Y, Z$, one of it multiplied by -1 , and use again the Jacobi identity to obtain

$$
0=2[J X, Y, Z]-2 J[X, Y, Z],
$$

whence $J[X, Y, Z]=[J X, Y, Z]=[X, J Y, Z]$, implying that the triple product is $J$-linear in the first two variables. (Note that the argument works for any endomorphism $J$ satisfying (S) and $J$-linearity of the LTS in the last variable.)

Proposition 1.2.4. If $J$ is an invariant complex structure on a LTS $\mathfrak{q}$, then the following are equivalent:

(i) $J$ is twisted.

(ii) $J$ is an automorphism of $\mathfrak{q}$, i.e.

$$
\forall X, Y, Z \in \mathfrak{q}: \quad[J X, J Y, J Z]=J[X, Y, Z] .
$$

(iii) $J$ is a derivation of $\mathfrak{q}$, i.e.

$$
\forall X, Y, Z \in \mathfrak{q}: \quad J[X, Y, Z]=[J X, Y, Z]+[X, J Y, Z]+[X, Y, J Z] .
$$

Proof. In view of the invariance, the equivalence of (i) and (iii) is clear. Using, in addition, that $J^{2}=-\mathrm{id}_{\mathfrak{q}}$, the equivalence of (i) and (ii) follows immediately.

Remark 1.2.5. Since the exponential of a derivation is an automorphism and $J=$ $e^{\frac{\pi}{2} J}$, one gets another proof of (iii) $\Rightarrow$ (ii).

1.3. Structure tensor and Hermitian Jordan triple products. Let $M=$ $G / H$ be a symmetric space with curvature tensor $R$ and invariant almost complex structure $\mathcal{J}$. For any $p \in M$, the space $\operatorname{End}\left(T_{p} M\right)$ of endomorphisms of the tangent space $T_{p} M$ splits into a direct sum of complex-linear and complex-antilinear (w.r.t. $\left.\mathcal{J}_{p}\right)$ elements. The endomorphism $R(X, Y)$ of $\mathfrak{X}(M)$ is by assumption complexlinear, but the endomorphism $R(X, \cdot) Z$ in general decomposes into two terms. We are thus lead to define the structure tensor $T$ of $\mathcal{J}$ by

$$
T(X, Y) Z=\frac{1}{2}\left(R(X, Y) Z-\mathcal{J} R\left(X, \mathcal{J}^{-1} Y\right) Z\right)=\frac{1}{2}(R(X, Y) Z+\mathcal{J} R(X, \mathcal{J} Y) Z) .
$$

Sometimes we will write $T(X, Y, Z)$ instead of $T(X, Y) Z$. Since $R$ and $\mathcal{J}$ are $G$ invariant, $T$ is a $G$-invariant tensor field of the same type as the curvature tensor. We write $J$ for $\mathcal{J}_{o}$. Then

$$
T_{o}(X, Y) Z=\frac{1}{2}\left([X, Y, Z]-J\left[X, J^{-1} Y, Z\right]\right)=\frac{1}{2}([X, Y, Z]+J[X, J Y, Z])
$$

is an $H$-equivariant map $\otimes^{3} \mathfrak{q} \rightarrow \mathfrak{q}$, called the structure tensor of $J$. Since $T_{o}$ and $R_{o}$ are, by invariance, equivalent to $T$ and $R$, we will suppress the index $o$ when there is no risk of confusion.

Proposition 1.3.1. (i) The structure tensor vanishes if and only if $J$ is straight.

(ii) The Lie algebra $\mathfrak{g}$ acts as a Lie algebra of derivations of $T$, and $\mathfrak{h}$ acts as a Lie algebra of derivations of $T_{o}$. 
(iii) For all vector fields $X, Y, Z$ on $M$,

$$
T(X, Y, Z)=T(Z, Y, X) .
$$

(iv) For all vector fields $X, Y, Z$ on $M$,

$$
T(X, Y, J Z)=-T(X, J Y, Z)=T(J X, Y, Z)=J T(X, Y, Z) .
$$

(v) $J$ is twisted if and only if for all vector fields $X, Y, Z$ on $M$,

$$
T(X, Y) Z-T(Y, X) Z=R(X, Y) Z,
$$

or, equivalently, $T_{o}(X, Y) Z-T_{o}(Y, X) Z=[X, Y, Z], X, Y, Z \in \mathfrak{q}$.

Proof. (i) This is clear from 1.2.3.

(ii) This is the infinitesimal version of the invariance under $G$, resp. $H$.

(iii) Using the Jacobi identity, we get

$$
\begin{aligned}
2(T(X, Y, Z)-T(Z, Y, X)) & =[X, Y, Z]-[Z, Y, X]+J([X, J Y, Z]-[Z, J Y, X]) \\
& =-[Z, X, Y]-J[Z, X, J Y]=0 .
\end{aligned}
$$

(iv) $T(X, Y) J Z=J T(X, Y) Z$ since $J$ is invariant;

$$
\begin{gathered}
T(X, J Y) Z=\frac{1}{2}([X, J Y, Z]-J[X, Y, Z])=-J T(X, Y) Z ; \\
T(J X, Y) Z=T(Z, Y) J X=J T(Z, Y) X=J T(X, Y) Z,
\end{gathered}
$$

using part (iii) twice.

(v) The tensor

$$
\begin{aligned}
T(X, Y) Z-T(Y, X) Z-[X, Y, Z] & =\frac{1}{2} J([X, J Y, Z]-[Y, J X, Z]) \\
& =\frac{1}{2} J([X, J Y, Z]+[J X, Y, Z])
\end{aligned}
$$

clearly vanishes if and only if $J$ is twisted.

Proposition 1.3.2. The structure tensor $T$ of an invariant twisted almost complex structure $\mathcal{J}$ on a symmetric space satisfies the following identities:

$$
\begin{gathered}
T(X, Y, Z)=T(Z, Y, X), \\
T(X, Y) \cdot T(U, V, W)=T(T(X, Y) U, V, W) \\
-T(U, T(Y, X) V, W)+T(U, V, T(X, Y) W) .
\end{gathered}
$$

Proof. The identity (JT1) has been proved above (Prop. 1.3.1 (iii)). We now

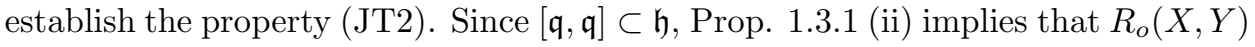
is a derivation of $T_{o}$. If $D$ is a derivation of $T_{o}$, then $J D$ is a skew-derivation of $T_{o}$ in the following sense: using 1.3.1 (iv) we get

$$
\begin{aligned}
J D T_{o}(X, Y, Z) & =J\left(T_{o}(D X, Y, Z)+T_{o}(X, D Y, Z)+T_{o}(X, Y, D Z)\right) \\
& =T_{o}(J D X, Y, Z)-T_{o}(X, J D Y, Z)+T_{o}(X, Y, J D Z) .
\end{aligned}
$$

Thus $2 T_{o}(X, Y) \in \operatorname{End}(\mathfrak{q})$ is the sum of the derivation $R_{o}(X, Y) \in \mathfrak{h}$ and the skewderivation $J R_{o}(X, J Y) \in \operatorname{End}(\mathfrak{q})$. Since $J$ is twisted, we obtain for the difference of these two elements:

$$
\frac{1}{2}\left(R_{o}(X, Y)-J R_{o}(X, J Y)\right)=-T_{o}(Y, X) .
$$


Using this, we can write (dropping the indices $o$ ):

$$
\begin{aligned}
T(X, Y) T(U, V, W)=\frac{1}{2}( & R(X, Y) \cdot T(U, V, W)+J R(X, J Y) \cdot T(U, V, W)) \\
=\frac{1}{2}( & T((R(X, Y)+J R(X, J Y)) U, V, W) \\
& \quad+T(U,(R(X, Y)-J R(X, J Y)) V, W) \\
& \quad+T(U, V,(R(X, Y)+J R(X, J Y)) W)) \\
= & T(T(X, Y) U, V, W)-T(U, T(Y, X) V, W) \\
& +T(U, V, T(X, Y) W) .
\end{aligned}
$$

This is the identity (JT2). Since both sides are $H$-invariant, this identity can be read as an identity for the whole tensor field $T$ and arbitrary vector fields.

Definition 1.3.3. A Jordan triple system (abbreviated JTS) is a vector space $V$ together with a trilinear map $T: V \times V \times V \rightarrow V,(X, Y, Z) \mapsto T(X, Y, Z)=$ $T(X, Y) Z$ satisfying the identities (JT1) and (JT2). A Hermitian JTS is a JTS T together with a complex structure $J$ on $V$ such that

$$
T(J X, Y, Z)=-T(X, J Y, Z)=T(X, Y, J Y)=J T(X, Y, Z)
$$

holds for all $X, Y, Z \in V$. Morphisms of (Hermitian) JTS are (complex) linear maps compatible with the respective triple products.

The preceding proposition together with 1.3.1 (iv) shows that the structure tensor of an invariant twisted almost complex structure is a field of Hermitian Jordan triple products.

Lemma 1.3.4. Let $T$ be a Jordan triple product defined on a vector space $\mathfrak{q}$. Then

$$
R_{T}(X, Y) Z:=T(X, Y) Z-T(Y, X) Z=T(X, Y) Z-T(Z, X) Y
$$

defines a Lie triple product on $\mathfrak{q}$.

Proof. Clearly $R_{T}$ is antisymmetric in the first two variables and satisfies the Jacobi identity (LT2). The identity (JT2) implies that $R_{T}(X, Y)$ is a derivation of $T$ and therefore also of $R_{T}$, whence (LT3).

We say that $R_{T}$ is the LTS associated to the JTS T, and if $R=R_{T}$, then we say that $T$ is a Jordan extension of the LTS R. It is clear that JTS-homomorphisms induce homomorphisms of associated LTS's. Therefore we call $T \mapsto R_{T}$ the (algebraic) Jordan-Lie functor. The Jordan-Lie functor is not bijective, but it does induce bijections of some important sub-categories.

Proposition 1.3.5. The Jordan-Lie functor induces a bijection between the category of Hermitian JTS's and the category of twisted complex LTS's. Its inverse is given by the formula

$$
T(X, Y) Z:=\frac{1}{2}\left(R(X, Y) Z-J R\left(X, J^{-1} Y\right) Z\right) .
$$

Proof. If $T$ is a Hermitian JTS, then $J$ is a twisted invariant complex structure for $R_{T}$ :

$$
\begin{gathered}
R_{T}(X, Y) J Z=T(X, Y, J Z)-T(Y, X, J Z)=J R_{T}(X, Y) Z \\
R_{T}(J X, Y)=T(J X, Y)-T(Y, J X)=-(T(X, J Y)-T(J Y, X))=-R_{T}(X, J Y) .
\end{gathered}
$$


Conversely, if $J$ is a twisted invariant complex structure for $R$, then, as seen above, the given formula defines a Hermitian JTS $T$. Thus both functors are well-defined. It is easily checked that they are inverse to each other: if $J$ is twisted invariant for $R$, then $R(X, J Y)$ is symmetric in $X$ and $Y$, and antisymmetrizing $T$ in the first two variables, we get back $R$. If $(T, J)$ is Hermitian, then

$$
\begin{aligned}
& R_{T}(X, Y) Z+J R_{T}(X, J Y) Z \\
& \quad=T(X, Y, Z)-T(Y, X, Z)+J(T(X, J Y, Z)-T(J Y, X, Z))=2 T(X, Y, Z) .
\end{aligned}
$$

Finally, homomorphisms in both categories are the same since compatibility with $R$ and $J$ is equivalent to compatibility with $T$ and $J$.

Theorem 1.3.6. The following three categories are equivalent:

(1) tcLTS: twisted complex Lie triple systems,

(2) hJTS: Hermitian Jordan triple systems,

(3) tcSS: germs of twisted complex symmetric spaces.

The functor tcSS $\rightarrow$ tcLTS is given by evaluating curvature tensor and almost complex structure at the base point, and the functor $t c S S \rightarrow$ hJTS by evaluating structure tensor and almost complex structure at the base point.

Proof. The equivalence of (1) and (2) has been established in the previous proposition. The equivalence of (1) and (3) follows immediately from Prop. 1.2.1.

Example 1.3.7 (The space of complex structures). Let

$$
N:=\left\{I \in \operatorname{Gl}(2 n, \mathbb{R}) \mid I^{2}=-\mathbf{1}_{2 n}\right\}
$$

be the space of complex structures on $V:=\mathbb{R}^{2 n}$ and choose a base point $I_{o}=$ $\left(\begin{array}{cc}0 & \mathbf{1}_{n} \\ -\mathbf{1}_{n} & 0\end{array}\right) \in N$. By linear algebra, the action of $\mathrm{Gl}(2 n, \mathbb{R})$ on $N$ by $g \cdot I=g I g^{-1}$ is transitive, and

$$
N=\operatorname{Gl}(2 n, \mathbb{R}) / \operatorname{Gl}(n, \mathbb{C})
$$

is a symmetric space. Let us show that it carries a natural twisted complex structure: the tangent space $T_{I_{o}} N$ is naturally identified with

$$
\mathfrak{q}:=\left\{X \in M(2 n, \mathbb{R}) \mid I_{o} X=-X I_{o}\right\}
$$

equipped with the Lie triple product

$$
[X, Y, Z]=[[X, Y], Z]=X Y Z+Z Y X-Z X Y-Y X Z
$$

induced from the ambient Lie algebra $\mathfrak{g l}(2 n, \mathbb{R})$. By a simple calculation one verifies that

$$
J: \mathfrak{q} \rightarrow \mathfrak{q}, \quad X \mapsto I_{o} X
$$

is an invariant twisted complex structure on $\mathfrak{q}$. The corresponding structure tensor is given by

$$
T(X, Y, Z)=\frac{1}{2}\left([X, Y, Z]+I_{o}\left[X, I_{o} Y, Z\right]\right)=X Y Z+Z Y X
$$


1.4. Invariant polarizations and Jordan pairs. Most of what has been said so far remains valid for tensor fields having the property $\mathcal{J}^{2}=$ id instead of $\mathcal{J}^{2}=-$ id.

Definition 1.4.1. An invariant polarization on a LTS $(\mathfrak{q}, R)$ is an endomorphism $J$ such that $J^{2}=\operatorname{id}_{\mathfrak{q}}$ and $[X, Y, J Z]=J[X, Y, Z]$. We call $J$ straight if $[J X, Y, Z]=$ $[X, J Y, Z]$ and twisted if $[J X, Y, Z]=-[X, J Y, Z](X, Y, Z \in \mathfrak{q})$. A polarized LTS is an LTS together with a twisted polarization. If the \pm 1 -eigenspaces of $J$ have the same dimension, then $J$ is also called a paracomplex structure.

As in the proof of Proposition 1.2.3 it is seen that straight polarized LTS are precisely the $J$-trilinear LTS, and these are nothing but direct products of two LTS. Analogously to Proposition 1.2.4, an invariant polarization is seen to be twisted if and only if it is a derivation. The structure tensor of $J$ is defined by

$$
T(X, Y) Z=\frac{1}{2}\left([X, Y, Z]-J\left[X, J^{-1} Y, Z\right]\right)=\frac{1}{2}([X, Y, Z]-J[X, J Y, Z]) .
$$

Then Propositions 1.3.1 and 1.3.2 and their proofs carry over to the polarized case without any changes, except that we have to replace "Hermitian Jordan triple systems" by "polarized Jordan triple systems" which are defined as Hermitian ones, but using a polarization instead of a complex structure. From a Jordan theoretic point of view, the important new feature is that polarized JTS are equivalent to Jordan pairs; cf. [Lo75] for the definition.

Theorem 1.4.2. The following four categories are equivalent:

(1) pLTS: polarized Lie triple systems,

(2) pJTS: polarized Jordan triple systems,

(3) JP: Jordan pairs,

(4) tpSS: (germs of) symmetric spaces with invariant twisted polarization.

The functor $t p S S \rightarrow p L T S$ is given by evaluating curvature tensor and involutive structure at the base point, and the functor tpSS $\rightarrow$ pJTS by evaluating structure tensor and involutive structure at the base point.

Proof. The equivalence of the categories (1), (2) and (4) is proved as in Th. 1.3.6, and the equivalence of (2) and (3) is proved in Lo75 p.10].

Example 1.4.3 (Space of polarizations). Let $I_{p, q}:=\left(\begin{array}{cc}\mathbf{1}_{p} & 0 \\ 0 & -\mathbf{1}_{q}\end{array}\right)$ be the base point in the space

$$
Y:=\left\{I \in \operatorname{Gl}(p+q, \mathbb{R}) \mid I^{2}=\mathbf{1}_{p+q}, \operatorname{sgn}(I)=(p, q)\right\}
$$

of polarizations on $V=\mathbb{R}^{p+q}$ with signature $(p, q)$. By calculations similar to those given in Example 1.3.5, we see that

$$
Y=\operatorname{Gl}(p+q, \mathbb{R}) /(\mathrm{Gl}(p, \mathbb{R}) \times \operatorname{Gl}(q, \mathbb{R}))
$$

is a symmetric space with invariant twisted para-complex structure. This structure is given by the map $J: X \mapsto I_{p, q} X$ on $\mathfrak{q}=\left\{\left(\begin{array}{cc}0 & A \\ B^{t} & 0\end{array}\right) \mid A, B \in M(p, q ; \mathbb{R})\right\}$, and both eigenspaces are of equal dimension, naturally isomorphic to $M(p, q ; \mathbb{R})$. All formulas remain valid if one replaces $\mathbb{R}$ by $\mathbb{C}$. The space $Y$ can be interpreted as the open dense subset of elements $(E, F)$ in the direct product $G r_{p, p+q} \times G r_{q, p+q}$ of Grassmannians such that $E \cap F=0$. 


\section{COMPLEXIFICATIONS AND SYMMETRIC SPACES WITH TWIST}

\subsection{Real forms and complexifications.}

Definition 2.1.1. (1) A global complexification of a symmetric space $M$ is a symmetric space $N$ together with an invariant almost complex structure $\mathcal{J}$ and a conjugation $\tau$ (i.e. an almost anti-holomorphic automorphism with $\tau^{2}=$ $\left.\operatorname{id}_{N}\right)$ such that $\tau(o)=o$ and the symmetric space $M$ is isomorphic to the $\tau$-fixed space $N^{\tau}$ (or to a union of connected components of $N^{\tau}$ ). In this situation we say that $M$ (or $\tau$ ) is a real form of $N$.

(2) A complexification of a germ $M^{o}$ of a symmetric space is defined as a germ $N^{o}$ of a symmetric space with invariant almost complex structure and germ $\tau$ of a conjugation such that $M^{o} \cong\left(N^{o}\right)^{\tau}$, and again $M^{o}$ is called a real form of $N^{o}$. The complexification is called straight, resp. twisted if $N^{o}$ is straight complex, resp. twisted complex.

(3) A morphism of complexifications is a morphism of germs of symmetric spaces with an extension to an almost-complex morphism of the complexifications.

(4) Replacing complex structures by para-complex structures, we get the corresponding notions of para-complexifications.

Definition 2.1.2. (1) A complexification of a LTS $(\mathfrak{q}, R)$ is a LTS $\mathfrak{p}$ with an invariant complex structure $J$ and a conjugation $\tau$ (i.e. a complex antilinear automorphism with $\left.\tau^{2}=\mathrm{id}_{\mathfrak{p}}\right)$ such that the LTS $(\mathfrak{q}, R)$ is isomorphic to the $\tau$-fixed LTS $\mathfrak{p}^{\tau}$. The complexification is called straight, resp. twisted if $\mathfrak{p}$ is straight complex, resp. twisted complex.

(2) A morphism of complexifications of LTS is a morphism of LTS together with a $\mathbb{C}$-linear extension to a morphism of the complexified LTS.

(3) Replacing complex structures by para-complex structures, we get the corresponding notions of para-complexifications.

Proposition 2.1.3. The category of local (para-)complexifications of germs of symmetric spaces is equivalent to the category of (para-) complexifications of Lie triple systems, and the subcategories of straight resp. twisted (para-)complexifications correspond to each other under this equivalence.

Proof. This is proved in the same way as Prop. 1.2.1.

Proposition 2.1.4. Any Lie triple product $R: \mathfrak{q} \times \mathfrak{q} \times \mathfrak{q} \rightarrow \mathfrak{q}$ has a unique straight complexification, given by the $\mathbb{C}$-trilinear extension $R_{\mathbb{C}}: \mathfrak{q}_{\mathbb{C}} \times \mathfrak{q}_{\mathbb{C}} \times \mathfrak{q}_{\mathbb{C}} \rightarrow \mathfrak{q}_{\mathbb{C}}$.

Proof. Because of Prop. 1.2.3, a straight complexification is necessarily given by $\mathbb{C}$-trilinear extension. In order to verify that this indeed defines a LTS, we express the identities (LT1), (LT2) and (LT3) by commutative diagrams of linear maps of some tensor products and apply to these the ordinary complexification functor for linear maps.

Note that, if $\mathfrak{q}$ is the -1-eigenspace of $\dot{\sigma}$ in $\mathfrak{g}$, then $\left(\mathfrak{q}_{\mathbb{C}}, R_{\mathbb{C}}\right)$ is the -1-eigenspace of the $\mathbb{C}$-linear extension $\dot{\sigma}_{\mathbb{C}}$ of $\sigma$ onto the complexified Lie algebra $\mathfrak{g}_{\mathbb{C}}$. Thus, if $M^{o}$ is the germ of the symmetric space $G / H$, then the germ $M_{\mathbb{C}}^{o}$ belonging to $\left(\mathfrak{q}_{\mathbb{C}}, R_{\mathbb{C}}\right)$ is the germ of $M_{\mathbb{C}}:=G_{\mathbb{C}} / H_{\mathbb{C}}$, where $G_{\mathbb{C}}$ is the connected simply connected group with Lie algebra $\mathfrak{g}_{\mathbb{C}}$ and $H_{\mathbb{C}}=G_{\mathbb{C}}^{\sigma}$ the group fixed under the unique holomorphic involution whose differential at the origin is $\dot{\sigma}_{\mathbb{C}}$. It is clear, however, that $M_{\mathbb{C}}$ is 
in general not a global complexification of $M=G / H$. Note that straight paracomplexifications always exist globally; they are just direct products of a space with itself.

\subsection{Twisted complexifications and Jordan extensions.}

Lemma 2.2.1. Let $\tau$ be a (para-)real form of a LTS $(\mathfrak{q}, R)$ with (para-)complex structure $J$. Then $\tau$ is an automorphism of the structure tensor $T(X, Y) Z=$ $\frac{1}{2}\left([X, Y, Z]-J\left[X, J^{-1} Y, Z\right]\right)$. In particular, the restriction $\left.T\right|_{\mathfrak{q}^{\tau}}$ of $T$ to $\mathfrak{q}^{\tau}$ is welldefined.

Proof.

$$
\begin{aligned}
\tau T(X, Y, Z) & =\frac{1}{2}\left(\tau[X, Y, Z]-\tau\left(J\left[X, J^{-1} Y, Z\right]\right)\right) \\
& =\frac{1}{2}\left([\tau X, \tau Y, \tau Z]-J\left[\tau X, J^{-1} \tau Y, \tau Z\right]\right) \\
& =T(\tau X, \tau Y, \tau Z),
\end{aligned}
$$

using twice that $J$ anticommutes with $\tau$.

Theorem 2.2.2. Let $(\mathfrak{q}, R)$ be a Lie triple system. The following objects are in one-to-one correspondence:

(1) twisted complexifications of $R$,

(2) twisted para-complexifications of $R$,

(3) Jordan-extensions $T$ of $R$ (i.e. Jordan triple systems $T$ such that $T(X, Y)-$ $T(Y, X)=R(X, Y))$.

Proof. The correspondences $(1) \rightarrow(3)$ and $(2) \rightarrow(3)$ are given by restricting the structure tensor of the complexified space to the real form; the preceding lemma shows that this is well-defined. Moreover, the structure tensor of a twisted (para)complex space is, according to Prop. 1.3.1 (v), a Jordan extension of the curvature tensor, and this relation carries over to real forms. In order to give the inverse constructions we have to establish first some basic results on Jordan triple systems.

Lemma 2.2.3. Let $T$ be a Jordan triple product on a vector space $V$ and $\alpha$ an endomorphism of $V$ with the property

$$
\forall x, y, z \in V: \quad T(\alpha x, y, \alpha z)=\alpha T(x, \alpha y, z) .
$$

Then the formula

$$
T^{\alpha}(x, y, z):=T(x, \alpha y, z)
$$

defines a Jordan triple product $T^{\alpha}$ on $V$.

Proof. The identity (JT2) for $T$ with $v$ and $y$ replaced by $\alpha v$ and $\alpha y$, respectively, yields

$$
\begin{aligned}
& T(T(u, \alpha v) x, \alpha y, z)-T(x, T(\alpha v, u) \alpha y, z) \\
& \quad+T(x, \alpha y, T(u, \alpha v) z)=T(u, \alpha v) T(x, \alpha y, z) .
\end{aligned}
$$

We apply $(*)$ to the middle triple product in the middle term and obtain

$$
\begin{aligned}
& T(T(u, \alpha v) x, \alpha y, z)-T(x, \alpha T(v, \alpha u) y, z) \\
& \quad+T(x, \alpha y, T(u, \alpha v) z)=T(u, \alpha v) T(x, \alpha y, z) .
\end{aligned}
$$

This is precisely the identity (JT2) for $T^{\alpha}$. Since $T^{\alpha}$ clearly satisfies (JT1), it is a Jordan triple product. 
Note that any involutive automorphism of $T$ satisfies $(*)$. An important application of the previous lemma is the existence of a canonical (para-)Hermitian complexification in the category of JTS's:

Theorem 2.2.5. Let $(V, T)$ be a Jordan triple system. We imbed $V$ into $\widetilde{V}:=$ $V \oplus V$ as the first factor and equip $\widetilde{V}$ with its canonical (para-)complex structure $J(x, y)=(-y, x)$ (resp. $I(x, y)=(y, x))$. Then there exist four Jordan triple systems $T_{\mathbb{C}}, T_{2}, T_{h \mathbb{C}}$ and $T_{p h \mathbb{C}}$ on $\widetilde{V}$ extending $T$ and uniquely determined by the following properties:

(i) $T_{\mathbb{C}}$ is $J$-linear in all three variables.

(ii) $T_{2}$ is I-linear in all three variables.

(iii) $T_{h \mathbb{C}}$ is Hermitian with respect to $J$.

(iv) $T_{p h \mathbb{C}}$ is polarized with respect to $I$.

Proof. Uniqueness is immediate from the definitions. We now prove existence: (i) is proved in the same way as Prop. 2.1.4. In order to prove (iii), one verifies that complex conjugation $\tau(x, y)=(x,-y)$ is an automorphism of $T_{\mathbb{C}}$. Since it is an involution, it satisfies condition $(*)$ of the lemma, and therefore

$$
T_{h \mathbb{C}}:=\left(T_{\mathbb{C}}\right)^{\tau}
$$

again is a JTS. It clearly is Hermitian and extends $T$. We now prove (ii): let $W^{ \pm}$be the eigenspaces of $I$; then $W^{+}$is the diagonal and $W^{-}$the antidiagonal, and $V \rightarrow$ $W^{ \pm}, v \mapsto \frac{1}{2}(v, \pm v)$ are vectorspace isomorphisms which we use as identifications in order to define the JTS $T_{2}:=T \oplus T$ on $W^{+} \oplus W^{-}$. It extends $T$ and is $I$ trilinear. This proves (ii). The para-conjugation $\tau(x, y)=(x,-y)$ is an involution of $T_{2}=T \oplus T$; in fact, in the coordinates $W^{+} \oplus W^{-}$it is just an exchange of the two factors. The lemma implies that $T_{p h \mathbb{C}}:=\left(T_{2}\right)^{\tau}$ is a JTS. It clearly is polarized by $I$ and extends $T$, thus (iv) is proved.

Now we finish the proof of Theorem 2.2.2 by constructing the correspondence $(3) \rightarrow(1)$ : let

$$
\widetilde{R}(X, Y):=R_{T_{h \mathbb{C}}}(X, Y)=T_{h \mathbb{C}}(X, Y)-T_{h \mathbb{C}}(Y, X) .
$$

Then $\widetilde{R}$ is twisted since $T_{h \mathbb{C}}$ is Hermitian (Prop. 1.3.4). Further, restriction of $\widetilde{R}$ to $\mathfrak{q}$ yields $R$ since the restriction of $T_{h \mathbb{C}}$ to $\mathfrak{q}$ is $T$, and $R_{T}=R$ by assumption. Therefore $\widetilde{R}$ is a twisted complexification of $R$.

The correspondence $(3) \rightarrow(2)$ is constructed in the same way, using this time the para-Hermitian complexification $T_{p h \mathbb{C}}$ of $T$. Finally, it is clear that these constructions are inverse to the ones given previously.

The theorem implies that the image of the Jordan-Lie functor $T \mapsto R_{T}$ is given by the LTS admitting a twisted (para-)complexification.

Definition 2.2.5. A (germ of a) symmetric space with twist is a (germ of a) symmetric space together with a Jordan extension $T$ of the curvature tensor $R$ (i.e. an invariant field of Jordan triple products such that $T(X, Y)-T(Y, X)=R(X, Y))$. Morphisms of (germs of) symmetric spaces with twist are morphisms of symmetric spaces which are compatible with the respective Jordan extensions.

Theorem 2.2.6. The following categories are equivalent:

(1) (real finite dimensional) Jordan triple systems. 
(2) germs of symmetric spaces with twist.

(3) germs of symmetric spaces with local twisted complexification.

(4) germs of symmetric spaces with local twisted para-complexification.

Proof. The bijection between the objects of the respective categories is given by Theorem 2.2.2 in combination with Prop. 2.1.3. It remains to be shown that all correspondences are functorial.

We show first that the constructions from Theorem 2.2.4 are functorial. If $\varphi$ : $V \rightarrow V^{\prime}$ is a homomorphism of JTS $T$ and $T^{\prime}$, then clearly $\varphi_{\mathbb{C}}$ is a homomorphism from $T_{\mathbb{C}}$ to $T_{\mathbb{C}}^{\prime}$. From this it follows that

$$
\varphi_{\mathbb{C}} T_{h \mathbb{C}}(u, v, w)=\varphi_{\mathbb{C}} T_{\mathbb{C}}(u, \bar{v}, w)=T_{\mathbb{C}}^{\prime}\left(\varphi_{\mathbb{C}} u, \varphi_{\mathbb{C}} \bar{v}, \varphi_{\mathbb{C}} w\right)=T_{h \mathbb{C}}\left(\varphi_{\mathbb{C}} u, \varphi_{\mathbb{C}} v, \varphi_{\mathbb{C}} w\right)
$$

since $\varphi \bar{v}=\overline{\varphi v}$. Thus $\varphi_{\mathbb{C}}$ is a homomorphism from $T_{h \mathbb{C}}$ to $T_{h \mathbb{C}}^{\prime}$. Similarly, for the para-complexifications.

Next we use the fact that the Jordan-Lie functor $T \mapsto R_{T}$ is functorial, i.e. we obtain homomorphisms of the twisted (para-)complex LTS equivalent to $T_{h \mathbb{C}}$ resp. $T_{p h \mathbb{C}}$.

Applying now Prop. 2.1.5, we can lift these homomorphisms on a local level, i.e. we have shown that $(1) \rightarrow(3)$ and $(1) \rightarrow(4)$ are functorial.

It is clear that restriction to a real form is functorial; thus $(3) \rightarrow(2)$ and $(4) \rightarrow$ (2) are functorial. It is also clear that evaluating at the base point is a functorial construction; thus $(2) \rightarrow(1)$ is functorial, and the theorem is proved.

Definition 2.2.7. The geometric Jordan-Lie functor is the forgetful-functor from the category of (germs of) symmetric spaces with twist to the category of (germs of) symmetric spaces.

The algebraic Jordan-Lie functor $T \mapsto R_{T}$ is the infinitesimal version of the geometric Jordan-Lie functor.

Remark 2.2.8. The Makarevič spaces introduced in [Be96b] and [Be98] are examples of symmetric spaces with twist: in fact, according to [Be96b, 2.3.3], the Makarevič spaces $X^{(\alpha)}$ defined there admit a global twisted or "Hermitian" complexification. The fact that they also admit globally a twisted para-complexification has been remarked in [Be98, 2.1, Ex.4]. The classification [Ma73 of irreducible Makarevič spaces essentially coincides with the classification of irreducible symmetric spaces with twist (Chapter 4); however, the close relation with Jordan triple systems and twisted complexifications was not remarked in Ma73.

2.3. Complexification functors and complexification diagrams. We write $\mathbb{C}, 2, h \mathbb{C}$ and $p h \mathbb{C}$ for the functors JTS $\rightarrow$ JTS defined in Theorem 2.2.4; we call them the functors of complexification, doubling, Hermitian complexification and twisted para-complexification in the category of Jordan triple systems.

Theorem 2.3.1. The category of germs of symmetric spaces with twist is stable under the functors $\mathbb{C}, 2, h \mathbb{C}$ and ph $\mathbb{C}$. Any two of the four functors commute, i.e. the opposite composition yields functors isomorphic to those obtained by the usual composition. Furthermore,

(i) $\mathbb{C} \circ \mathbb{C}=2 \circ \mathbb{C}, h \mathbb{C} \circ h \mathbb{C}=2 \circ h \mathbb{C}, p h \mathbb{C} \circ p h \mathbb{C}=2 \circ p h \mathbb{C}$,

(ii) $\mathbb{C} \circ h \mathbb{C} \cong \mathbb{C} \circ p h \mathbb{C} \cong h \mathbb{C} \circ p h \mathbb{C}$,

(iii) $p h \mathbb{C} \circ h \mathbb{C} \circ \mathbb{C} \cong 2 \circ h \mathbb{C} \circ \mathbb{C}$. 
Proof. Since the functors $\mathbb{C}, 2, h \mathbb{C}$ and $p h \mathbb{C}$ are functors in the category of Jordan triple systems, they are by Theorem 2.2.6 also functors in the category of germs of symmetric spaces with twist.

The "commutation relations" (i)-(iii) are verified by elementary calculations, using the "multiplication table" for the four kinds of structures: the product of two commuting complex structures is para-complex; the product of two commuting twisted structures is straight, and so on.

Note that exactly one interesting new functor arises by composition: it is a sort of double complexification-functor $\mathbb{C} \circ h \mathbb{C} \cong \mathbb{C} \circ p h \mathbb{C} \cong h \mathbb{C} \circ p h \mathbb{C}$ in the category of symmetric spaces with twist. It assigns to a space another one having four times its real dimension and carrying two commuting almost complex structures. We thus have four non-trivial complexification-functors $\mathbb{C}, h \mathbb{C}, p h \mathbb{C}$ and $\mathbb{C} \circ h \mathbb{C}$ which are related by (i)-(iii). The situation may be visualized by the complexification diagram of a symmetric space with twist $T$ :

$$
M \stackrel{\nearrow}{\longrightarrow} M_{\mathbb{C}} \quad M_{h \mathbb{C}} \rightarrow\left(M_{h \mathbb{C}}\right)_{\mathbb{C}}
$$

Example 2.3.2. Using the notation of Example 1.3.7, let $s(z)=\bar{z}$ be the standard complex conjugation of $\mathbb{R}^{2 n}=\mathbb{C}^{n}$. Then $\tau(I):=-s I s$ defines a real form of $N$ fixing the base point $I_{o}$. The group commuting with $s=\left(\begin{array}{cc}\mathbf{1} & 0 \\ 0 & -1\end{array}\right)$ is isomorphic to $\operatorname{Gl}(n, \mathbb{R}) \times \operatorname{Gl}(n, \mathbb{R})$, and

$$
N^{\tau}=(\mathrm{Gl}(n, \mathbb{R}) \times \mathrm{Gl}(n, \mathbb{R})) / \mathrm{Gl}(n, \mathbb{R})
$$

is the general linear group, viewed as a symmetric space. Thus, with the Jordan extension $T(X, Y, Z)=X Y Z+Z Y X$ of $\mathfrak{g l}(n, \mathbb{R})$, the complexification diagram of $M=\mathrm{Gl}(n, \mathbb{R})$ reads

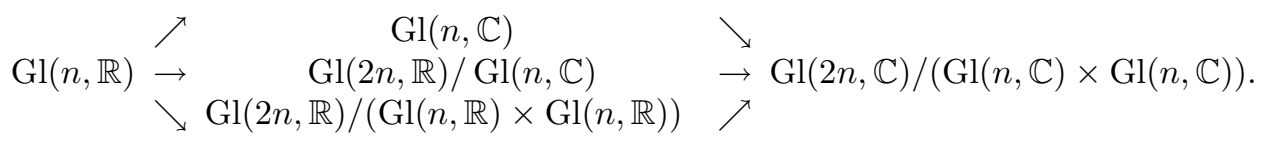

\section{Classification of (Para-)Complex structures}

It is rather easy to deduce from the preceding results equivalences of categories between "regular" spaces and the corresponding "regular" algebraic structures. For instance, the existence of invariant compatible (pseudo-)Riemannian tensor fields translates to the existence of associative bilinear forms on Jordan triple systems, and as for general symmetric spaces, semisimplicity is related to non-degeneracy of trace-forms (the correspondence between bounded symmetric domains and positive Hermitian JTS is contained as a special case). We refer to [Be99] for the details and content ourselves here with the discussion of the simple spaces; among other results we prove that invariant (para-)complex structures on a simple symmetric space are either straight or twisted.

Recall that ideals in triple systems are kernels of homomorphisms, and triple systems are called simple if their dimension is bigger than one and they contain no proper ideals. Simple Lie triple systems correspond to germs of irreducible symmetric spaces. A LTS $\mathfrak{q}$ is called semisimple if its Ricci-form

$$
(u, v) \mapsto \operatorname{tr}([u, \cdot, v])
$$


is non-degenerate. Semisimple LTS correspond to germs of semisimple symmetric spaces, and the Ricci-form of the LTS is the usual Ricci-form, evaluated at the base point. It is known that a simple JTS is semisimple and that a semisimple LTS has a unique decomposition into simple ideals (cf. Lo69]).

For a LTS $\mathfrak{q}$ and $\mathfrak{h}:=[\mathfrak{q}, \mathfrak{q}] \subset \operatorname{Der}(\mathfrak{q})$, we denote by

$$
\operatorname{End}(\mathfrak{q})^{\mathfrak{h}}=\{X \in \operatorname{End}(\mathfrak{q}) \mid \forall Y \in \mathfrak{h}:[Y, X]=0\}
$$

the algebra of $\mathfrak{h}$-invariants in $\operatorname{End}(\mathfrak{q})$. If $\mathfrak{q}$ is semisimple, we denote by $\operatorname{End}(\mathfrak{q})=$ $\operatorname{Sym}(\mathfrak{q}) \oplus \operatorname{Asym}(\mathfrak{q})$ the decomposition of $\operatorname{End}(\mathfrak{q})$ into spaces of symmetric and skewsymmetric operators w.r.t. the Ricci-form. Since the Ricci-form is $\mathfrak{h}$-invariant, the projectors onto $\operatorname{Sym}(\mathfrak{q})$ resp. $\operatorname{Asym}(\mathfrak{q})$ are $\mathfrak{h}$-equivariant, and therefore we have a decomposition

$$
\operatorname{End}(\mathfrak{q})^{\mathfrak{h}}=\operatorname{Sym}(\mathfrak{q})^{\mathfrak{h}} \oplus \operatorname{Asym}(\mathfrak{q})^{\mathfrak{h}} .
$$

Theorem 3.1. If $\mathfrak{q}$ is a simple LTS, then precisely the following five cases can arise:

1. generic

2. straight complex generic

3. twisted complex generic

4. twisted polarized generic

5. all structures exist

$\begin{array}{lll}\operatorname{End}(\mathfrak{q})^{\mathfrak{h}} & \operatorname{Sym}(\mathfrak{q})^{\mathfrak{h}} & \operatorname{Asym}(\mathfrak{q})^{\mathfrak{h}} \\ \mathbb{R} & \mathbb{R} & 0 \\ \mathbb{C} & \mathbb{C} & 0 \\ \mathbb{C} & \mathbb{R} & \mathbb{R} \\ \mathbb{R} \times \mathbb{R} & \mathbb{R} & \mathbb{R} \\ \mathbb{C} \times \mathbb{C} & \mathbb{C} & \mathbb{C}\end{array}$

In this table, the isomorphy class of $\operatorname{End}(\mathfrak{q})^{\mathfrak{h}}$ as an associative algebra is given, and $\operatorname{Sym}(\mathfrak{q})^{\mathfrak{h}}$ and $\operatorname{Asym}(\mathfrak{q})^{\mathfrak{h}}$ are described as vector spaces (and as sub-JTS of the JTS $\left.\operatorname{End}(\mathfrak{q})^{\mathfrak{h}}\right)$. By "generic" we mean that the LTS $\mathfrak{q}$ has no additional straight or twisted structure besides the one mentioned.

Proof. Step 1. We denote by $g(u, v)=\operatorname{tr}([u, \cdot, v])$ the Ricci-form of $\mathfrak{q}$. Since $g$ is non-degenerate, the identity

$$
g(R(x, y) v, w)=g(R(v, w) x, y)
$$

holds (cf. [Hel62, p. 68] or [Lo69, p. 146]). Now let $X \in \operatorname{Sym}(\mathfrak{q})^{\mathfrak{h}}$. Using (3.2) twice, we have for all $a, b, u, v \in \mathfrak{q}$,

$$
g(R(X a, b) u, v)=g(R(u, v) X a, b)=g(R(u, v) a, X b)=g(R(a, X b) u, v),
$$

and therefore $R(X a, b)=R(a, X b)$ since $g$ is non-degenerate. This together with the fact that $R$ is $X$-linear in the last variable implies that (cf. the proof of Prop.

$$
R(X a, b)=R(a, X b)=X R(a, b),
$$

i.e. the Lie triple product on $\mathfrak{q}$ is $X$-trilinear. Next we complexify the whole set-up, thus assuring the existence of eigenvalues of $X$. The eigenspaces of $X_{\mathbb{C}}$ are ideals of the LTS $\mathfrak{q}_{\mathbb{C}}$ since $R_{\mathbb{C}}$ is $X_{\mathbb{C}}$-trilinear. Now we have to distinguish two cases:

(a) The LTS $\mathfrak{q}_{\mathbb{C}}$ is simple. Then $X$ has just one eigenvalue $t \in \mathbb{C}$, and the corresponding eigenspace must be all of $\mathfrak{q}$, i.e. $X=t \mathrm{id}_{\mathfrak{q}_{\mathbb{C}}}$. It follows that $t$ must have been already real. We have shown that in case (a) $\operatorname{Sym}(\mathfrak{q})^{\mathfrak{h}}=\mathbb{R} \mathrm{id}_{\mathfrak{q}}$.

(b) The LTS $\mathfrak{q}_{\mathbb{C}}$ is not simple. As in the case of Lie algebras, this means that $\mathfrak{q}$ is already a simple complex LTS, and $\mathfrak{q}_{\mathbb{C}} \cong \mathfrak{q} \oplus \mathfrak{q}$. Then the arguments from case (a) show that $\operatorname{Sym}(\mathfrak{q})^{\mathfrak{h}}=\mathbb{C}_{i_{\mathfrak{q}}}$. 
Step 2. If $\operatorname{Asym}(\mathfrak{q})^{\mathfrak{h}}=0$, then $\operatorname{End}(\mathfrak{q})^{\mathfrak{h}}=\operatorname{Sym}(\mathfrak{q})^{\mathfrak{h}}$, and we are in cases 1 or 2 of the table. In the first case there exists no invariant almost (para-)complex structure, and in the second case there exist precisely two invariant almost complex structures which are straight.

We now assume that $\operatorname{Asym}(\mathfrak{q})^{\mathfrak{h}} \neq 0$ and let $0 \neq X \in \operatorname{Asym}(\mathfrak{q})^{\mathfrak{h}}$. The arguments used in the beginning of Step 1 show that for all $u, v \in \mathfrak{q}$,

$$
R(X u, v)=-R(u, X v) .
$$

It follows that $R(X u, v) w+R(u, X v) w+R(u, v) X w=X R(u, v) w$, i.e. $X$ is a derivation of $\mathfrak{q}$. Since $\mathfrak{q}$ is simple, all derivations are inner, i.e. $\mathfrak{h}=\operatorname{Der}(\mathfrak{q})(\mathrm{cf}$. Lo69]), and thus $X \in \mathfrak{h}$. By assumption, $X$ commutes with $\mathfrak{h}$, therefore $X \in \mathfrak{z}(\mathfrak{h})$. Decomposing $\mathfrak{q}$ into a direct sum $\mathfrak{q}=\bigoplus_{i} \mathfrak{q}_{i}$ of irreducible $\mathfrak{h}$-modules, we see that the restriction of $X$ to $\mathfrak{q}_{i}$ is either zero or bijective. Then the same is true for the element $X^{2}$ of $\operatorname{Sym}(\mathfrak{q})$. But according to Step $1, X^{2} \in \operatorname{Sym}(\mathfrak{q})^{\mathfrak{h}}$ operates as a (real or complex) scalar $t \mathrm{id}_{\mathfrak{q}}$ on $\mathfrak{q}$; it follows that either $X=0$ or $X^{2}=t \mathrm{id}_{\mathfrak{q}} \neq 0$. The former case being excluded, we conclude that $X$ is bijective, and since it commutes with all elements of $\operatorname{End}(\mathfrak{q})^{\mathfrak{h}}$, the map

$$
\operatorname{Sym}(\mathfrak{q})^{\mathfrak{h}} \rightarrow \operatorname{Asym}(\mathfrak{q})^{\mathfrak{h}}, \quad Y \mapsto X Y=Y X
$$

is a bijection. Therefore only the cases that both are isomorphic to $\mathbb{R}$ or that both are isomorphic to $\mathbb{C}$ can appear. If both are isomorphic to $\mathbb{R}$, we can rescale $X$ such that $X^{2}=\mathrm{id}_{\mathfrak{q}}$ or $X^{2}=-\mathrm{id}_{\mathfrak{q}}$. If $X^{2}=\mathrm{id}$, then (3.4) shows that it is an invariant twisted polarization, and we are in case 4 of the claim. If $X^{2}=-\mathrm{id}_{\mathfrak{q}}$, then we are in case 3 of the claim. Finally, if both $\operatorname{Sym}(\mathfrak{q})^{\mathfrak{h}}$ and $\operatorname{Asym}(\mathfrak{q})^{\mathfrak{h}}$ are isomorphic to $\mathbb{C}$, then we can rescale $X$ by a complex scalar such that $X^{2}=\mathrm{id}_{\mathfrak{q}}$, and thus $X$ is an invariant twisted polarization on a straight complex LTS, and we are in case 5 of the claim.

Corollary 3.2. If $\mathfrak{q}$ is a simple LTS, then either $\mathfrak{q}$ is a simple $\mathfrak{h}$-module or it is the direct sum of two irreducible $\mathfrak{h}$-modules which are dual to each other. The latter case arises if and only if $\mathfrak{q}$ is twisted polarized, and then the irreducible $\mathfrak{h}$-modules are the eigenspaces of the invariant polarization.

Proof. By the converse of Schur's lemma, $\mathfrak{q}$ is irreducible if $\operatorname{End}(\mathfrak{q})^{\mathfrak{h}}$ is a field. This happens precisely in cases $1-3$ of the preceding theorem, and in the other cases the determination of $\operatorname{End}(\mathfrak{q})^{\mathfrak{h}}$ shows that there are precisely two irreducible submodules. They are dual w.r.t. the Ricci-form; in fact, the eigenspaces of the invariant polarization are maximal isotropic complementary subspaces w.r.t. the Ricci-form, and therefore the Ricci-form sets up a duality between them.

Corollary 3.3. If $\mathfrak{q}$ is a simple LTS, then an invariant complex structure on $\mathfrak{q}$ is either straight or twisted.

Proof. In cases 1 and 4 of Theorem 3.1, there are no invariant almost complex structures; in cases 2 and 3 there are precisely two, and they are straight in case 2 and twisted in case 3 ; in case 5 there are four invariant almost complex structures, and two of them are straight and two are twisted.

Corollary 3.4. If $\mathfrak{q}$ is a simple LTS, then the representation $\mathfrak{h} \rightarrow \mathfrak{g l}(\mathfrak{q})$ is never quaternionic. 
Proof. The claim means just that $\operatorname{End}(\mathfrak{q})^{\mathfrak{h}}$ is under the given assumptions never isomorphic to $\mathbb{H}$. This follows immediately from the table in Theorem 3.1.

Corollary 3.5. Let $M$ be an irreducible symmetric space.

(i) If $M$ is not straight complex, the Ricci-form is (up to a real scalar) the only invariant pseudo-Riemannian metric on $M$. If $M$ is straight complex, then all invariant pseudo-Riemannian metrics on $M$ are of the form $(X, Y) \mapsto$ $\operatorname{tg}\left(e^{\varphi \mathcal{J}} X, Y\right)$ with $t, \varphi \in \mathbb{R}$, where $g$ is the Ricci-form and $\mathcal{J}$ the straight invariant almost complex structure.

(ii) $M$ admits an invariant symplectic form $\omega$ if and only if it is twisted complex or twisted polarized. This form satisfies $\mathrm{d} \omega=0$ (i.e. $M$ is pseudo-Kähler, resp. para-Kähler).

Proof. The Ricci-form, being non-degenerate, sets up an $\mathfrak{h}$-equivariant bijection

$$
b: \operatorname{End}(\mathfrak{q}) \rightarrow \operatorname{Bil}(\mathfrak{q}):=(\mathfrak{q} \otimes \mathfrak{q})^{*}, \quad X \mapsto \operatorname{Ric}_{o}(X \cdot, \cdot)
$$

such that $\operatorname{Sym}(\mathfrak{q})$ corresponds to symmetric and Asym(q) to skew-symmetric bilinear forms on $\mathfrak{q}$. Moreover $b(X)$ is non-degenerate iff $X$ is non-singular. Under this correspondence, part (i) and the first claim of (ii) follow directly from Theorem 3.1. For the last claim, recall that for any $p$-form $\omega$, the exterior differential $\mathrm{d} \omega$ is given by anti-symmetrizing $\nabla \omega$. We apply this to the invariant 2 -form $\omega$ equivalent to $\operatorname{Ric}(\mathcal{J} \cdot, \cdot)$ where $\mathcal{J}$ is twisted. Then, by invariance, $\nabla \omega=0$, and therefore the three-form $\mathrm{d} \omega$ obtained by antisymmetrizing this tensor field also vanishes.

\section{Classification of Symmetric Spaces With twist}

The purpose of this chapter is to give a complete list of simple non-exceptional symmetric spaces with twist and to specify for each such space structure tensor and complexification diagram. Since there are nearly one-hundred series of simple spaces, we will not write down all complexification diagrams as in Example 2.3.2, but we organize our list in the following way:

- Table 4.2.1: first we list spaces having invariant structures of all kinds (complex and para-complex, straight and twisted). They have been classified in various ways, cf. [KanKo85, p. 97] for a Lie theoretic approach and [Lo75] for a Jordan theoretic approach. The complexification diagram of such a space is rather trivial: it contains only direct products.

- Table 4.2.2: second we list straight real forms of the spaces from Table 4.2.1; they have only a twisted para-complex structure. As for 4.2.1, their classification is well-known. For convenience to the reader, we include in Tables 4.2.2 and 4.2.1, also exceptional cases; cf. [KanKo85]. The complexification diagrams are easy to calculate: straight and Hermitian complexification are isomorphic, and the double complexification reduces to a direct product of straight complexifications.

- Table 4.2.3: third we list para-real forms of spaces from Table 4.2.1 which are twisted complex (in fact, pseudo-Hermitian). As above, their complexification diagram is easily calculated since now twisted para- and straight complexification are isomorphic.

- Table 4.2.4: fourth we list para-real forms of spaces from Table 4.2.1 which are complex symmetric spaces. Again, the complexification diagram is easily determined. 
- Table 4.2.5: finally, we list para-real forms of spaces from Table 4.2.2 (on the left in the examples). This is the "generic" case, having non-trivial complexification diagrams which have to be calculated case-by-case.

The para-real forms appearing in the last three tables are determined by using the following lemma:

Lemma 4.0. For a given JTS T, all para-real forms of $T_{p h \mathbb{C}}$ are of the form

$$
T^{\alpha}(X, Y, Z)=T(X, \alpha Y, Z)
$$

for a suitable $\alpha$, satisfying condition $(*)$ of Lemma 2.2.3.

Proof. If $\nu$ is a para-conjugation of $T_{p h \mathbb{C}}$, we write $\nu=\tau \beta$ where $\tau$ is the standardconjugation. Then, since $\beta \circ J=J \circ \beta, \beta$ preserves the eigenspaces of $J$ and can be written in the form $\beta=\left(\alpha_{+}, \alpha_{-}\right)$. The condition id $=\nu^{2}=\tau \beta \tau \beta$ reads $\alpha_{+}=\alpha_{-}^{-1}$ because $\tau$ exchanges the eigenspaces. Let $\alpha:=\alpha_{+}$. Then a short calculation shows that the condition that $\left(\alpha, \alpha^{-1}\right)$ be an automorphism of $T_{p h \mathbb{C}}$ is equivalent to condition $(*)$ of Lemma 2.2.3.

We call the variety of invertible endomorphisms $\alpha$ satisfying condition $(*)$ of Lemma 2.2.3 the structure variety. It can be shown that the isomorphism class of $T^{\alpha}$ depends only on the connected component of the structure variety belonging to $\alpha$ (cf. Be96b, Lemma 2.2.11]). Thus our strategy is to determine representatives of the connected component of the structure variety. For JTS belonging to simple Jordan algebras this has been carried out (although stated without proof) by B.O. Makarevič (Ma73]). However, comparison with the classification of simple real JTS by E. Neher ([N80], [N81]) shows that his list is (up to some minor errors) complete. The method used by E. Neher is different from the one explained above.

\subsection{Algebraic part: classification of simple Jordan triple systems.}

4.1.0. Notation. The space of $p \times q$-matrices over $\mathbb{F}=\mathbb{R}, \mathbb{C}$ or $\mathbb{H}$ is denoted by $M(p, q ; \mathbb{F})$, and we let $M(n, \mathbb{F}):=M(n, n ; \mathbb{F})$. If $A$ is an invertible $n \times n$-matrix over $\mathbb{F}$ and $\varepsilon$ an anti-automorphism of $\mathbb{F}$ we denote by

$$
\operatorname{Herm}(A, \varepsilon, \mathbb{F}):=\left\{X \in M(n, \mathbb{F}) \mid A^{-1} X^{t} A=\varepsilon X \varepsilon^{-1}\right\}
$$

the corresponding space of Hermitian matrices. The corresponding space of skewHermitian matrices is denoted by $\operatorname{Aherm}(A, \varepsilon, \mathbb{F})$. If $\mathbb{F}=\mathbb{R}$ or $\mathbb{C}$ and $\varepsilon=\mathrm{id}_{\mathbb{F}}$, one writes instead of Herm and Aherm also Sym and Asym, and if $\mathbb{F}=\mathbb{C}$ or $\mathbb{H}$ and $\varepsilon(w)=\bar{w}$ is the standard-conjugation, one drops the parameter $\varepsilon$ in the notation. A second conjugation of $\mathbb{H}$ is given by the composition $\varphi$ of the standard conjugation with conjugation by the quaternion $j$. Then $\operatorname{Herm}(I, \varphi, \mathbb{H})$ and $\operatorname{Aherm}(I, \mathbb{H})$ are isomorphic (cf. Be96b, 1.2.1]). If $A=I_{n}$, we just note the $\operatorname{rank} n$ instead of $A$, and if $A=I_{p, q}=\left(\begin{array}{cc}\mathbf{1}_{p} & 0 \\ 0 & -\mathbf{1}_{q}\end{array}\right)$, we note the signature $(p, q)$ instead of $A$. If not otherwise specified, $p, q, m, k, l$ are integers such that $n=2 m, p+q=n(p>q), k+l=p$. We frequently use the matrix $F:=F_{m}:=\left(\begin{array}{cc}0 & \mathbf{1}_{m} \\ -\mathbf{1}_{m} & 0\end{array}\right)$.

4.1.1. Classification of simple complex Jordan pairs (cf. [Lo77, 4.11]). These are para-complexifications of complex Jordan triple systems $T$ defined on a complex vector space $V_{\mathbb{C}}$. We specify $V_{\mathbb{C}}$ and a (non-unique, but natural) choice for $T$. For type 4 we use the notation $x y=(x \mid e) y+(y \mid e) x-(x \mid y) e$, where $e$ is the first canonical basis vector in $\mathbb{C}^{n}$ and $(u \mid v)=\sum_{i} u_{i} v_{i}$. 


$$
\begin{array}{lll}
\multicolumn{2}{l}{\text { underlying space } V_{\mathbb{C}}} & \text { Jordan triple product on } V_{\mathbb{C}} \\
\text { 1. } & M(n, \mathbb{C}) & T(X, Y, Z)=\frac{1}{2}(X Y Z+Z Y X) \\
\text { 2. } & \operatorname{Sym}(n, \mathbb{C}) & T(X, Y, Z)=\frac{1}{2}(X Y Z+Z Y X) \\
\text { 3. } & \operatorname{Sym}(F, \mathbb{C})(\cong \operatorname{Asym}(2 m, \mathbb{C})) & T(X, Y, Z)=\frac{1}{2}(X Y Z+Z Y X) \\
\text { 4. } & \mathbb{C}^{n} & T(x, y, z)=x(y z)-y(x z)+(x y) z \\
\text { 5. } & \operatorname{Herm}\left(3, \mathbb{O}_{\mathbb{C}}\right) & T(X, Y, Z)=\frac{1}{2}(X Y Z+Z Y X) \\
6 . & M(p, q ; \mathbb{C}), p>q & T(X, Y, Z)=\frac{1}{2}\left(X Y^{t} Z+Z Y^{t} X\right) \\
\text { 7. } & \operatorname{Asym}(2 m+1, \mathbb{C}) & T(X, Y, Z)=\frac{1}{2}(X Y Z+Z Y X) \\
\text { 8. } & M\left(1,2 ; \mathbb{O}_{\mathbb{C}}\right) & T(X, Y, Z)=\frac{1}{2}(X Y Z+Z Y X)
\end{array}
$$

The first five pairs are of the first kind: they have invertible elements and thus come from Jordan algebras. The Jordan algebra structure is given by $X \cdot Y=$ $\frac{1}{2}(X Y+Y X)$ in cases $1,2,3$ and 5 and by $x y$ in case 4 . The other pairs do not have invertible elements, but we have natural embeddings into Jordan algebras: 7 $\rightarrow 1$ is clear; $6 \rightarrow 2$ is of the form $X \mapsto\left(\begin{array}{cc}0 & X^{t} \\ X & 0\end{array}\right)$ and $8 \rightarrow 5$ of the form $X \mapsto\left(\begin{array}{cc}0 & \bar{X}^{t} \\ X & 0\end{array}\right)$.

4.1.2. Classification of real Jordan pairs. Real forms of the above complex Jordan pairs are given by conjugations $\tau$ of the complex JTS $\left(V_{\mathbb{C}}, T\right)$ specified above. We indicate the space and the corresponding conjugation $\tau$; the JTS and with it the Jordan pair structure is given by restricting the triple product from the corresponding complex space.

$$
\begin{aligned}
& \text { real form } V=V_{\mathbb{C}}^{\tau} \\
& \text { 1.1. } \operatorname{Herm}(n, \mathbb{C}) \\
& \text { 1.2. } M(n, \mathbb{R}) \\
& \text { 1.3. } n=2 m: M(m, \mathbb{H}) \\
& \text { 2.1. } \operatorname{Sym}(n, \mathbb{R}) \\
& \text { 2.2. } n=2 m: \operatorname{Herm}(m, \varphi, \mathbb{H}) \\
& \text { conjugation } \tau \text { of } V_{\mathbb{C}} \\
& \tau(Z)=\bar{Z}^{t} \\
& \tau(Z)=\bar{Z} \\
& \tau(Z)=F \bar{Z} F^{-1} \\
& \tau(Z)=\bar{Z} \\
& \tau(Z)=F \bar{Z} F^{-1} \\
& \text { 3.1. } \operatorname{Herm}(m, \mathbb{H}) \\
& \tau(Z)=\bar{Z}^{t}=F \bar{Z} F^{-1} \\
& \text { 3.2. } \operatorname{Sym}(F, \mathbb{R})(\cong \operatorname{Asym}(2 m, \mathbb{R})) \quad \tau(Z)=\bar{Z} \\
& \text { 4.1. } \quad \mathbb{R}^{p} \times \mathbb{R}^{q}, p+q=n, p \geq 1 \quad \tau(z)=\bar{x}-\bar{y}\left(z=x+y, x \in \mathbb{C}^{p}, y \in \mathbb{C}^{q}\right) \\
& \text { 5.1. } \operatorname{Herm}(3, \mathbb{O}) \quad \tau(Z)=\bar{Z} \\
& \text { 5.2. } \operatorname{Herm}\left(3, \mathbb{O}_{s}\right) \quad \tau(Z)=\widetilde{Z} \\
& \text { 6.1. } \quad M(p, q ; \mathbb{R}), p>q \quad \tau(Z)=\bar{Z} \\
& \text { 6.2. } p=2 r, q=2 s: M(r, s ; \mathbb{H}) \quad \tau(Z)=F_{r} \bar{Z} F_{s}^{-1} \\
& \text { 7.1. } \operatorname{Asym}(2 m+1, \mathbb{R}) \quad \tau(Z)=\bar{Z} \\
& \text { 8.1. } M(1,2 ; \mathbb{O}) \quad \tau(Z)=\bar{Z} \\
& \text { 8.2. } M\left(1,2 ; \mathbb{O}_{s}\right) \quad \tau(Z)=\widetilde{Z}
\end{aligned}
$$

Construction of Jordan triple systems from Jordan pairs. To each polarized JTS $T_{p h \mathbb{C}}$ with $T$ from one of the tables above we determine all para-real forms $T^{\alpha}$ by giving representatives $\alpha$ of the structure variety of $T$ as explained after Lemma 4.0. 
c-duality. On the level of LTS and JTS, the well-known duality between compact and non-compact symmetric spaces generalizes to the duality given by the functor $R \mapsto-R, T \mapsto-T$. It is clear that $T^{-\alpha}=-T^{\alpha}$. We just list one of the endomorphisms $\alpha$ and $-\alpha$, but in general there is no canonical choice between them. However, if $T$ comes from a Jordan algebra, in many cases it is possible to choose $\alpha$ to be a Jordan algebra automorphism; then $-\alpha$ is just an automorphism of triple systems, but not of algebras, and the choice becomes canonical. If $T^{\alpha}$ is straight complex or twisted polarized, then $T^{\alpha}$ and $-T^{\alpha}$ are always isomorphic, where the isomorphism is given by the invariant (para-)complex structure $J$.

\subsubsection{Complex-antilinear modifications of complex Jordan pairs.}

1.A. $\alpha(X)=\bar{X}$

1.B. $\quad \alpha(X)=I_{p, q} \bar{X}^{t} I_{u, v}$

2.A. $\alpha(X)=I_{p, q} \bar{X} I_{p, q}$

3.A. $\quad \alpha(X)=F I_{p, q} \bar{X}^{t} F I_{p, q}(p+q=2 m)$

4.A. $\alpha(z)=I_{p, q} \bar{z}$

4.B. $(n$ even $) \quad \alpha(z)=F \bar{z}$

6.A. $\quad \alpha(X)=I_{k, l} \bar{X} I_{i, j}(k+l=p, i+j=q)$

7.A. $\quad \alpha(X)=I_{p, q} \bar{X} I_{p, q}(p+q=2 m+1)$

4.1.4. Complex-linear modifications of complex Jordan pairs.

1.a. $\quad \alpha=$ id

1.b. $\quad \alpha(X)=X^{t}$

1.c. $n=2 m: \quad \alpha(X)=F X^{t} F^{-1}$

2.a. $\alpha=$ id

2.b. $n=2 m: \quad \alpha(X)=F X F^{-1}$

3.a. $\quad \alpha=$ id

3.b. $\alpha(X)=F X F^{-1}$

4.a. $\quad \alpha=I_{q+1, p-1}, p>0$

6.a. $\alpha=$ id

6.b. $(p=2 r, q=2 s): \quad \alpha(X)=F_{r} X F_{s}^{-1}$

7.a. $\quad \alpha=$ id

4.1.5. Modifications of real (non-complex) Jordan pairs.

1.1.a. $\quad \alpha(X)=I_{p, q} X I_{p, q}$

1.1.b. $\alpha(X)=X^{t}$

1.1.c. $n=2 m: \quad \alpha(X)=F X^{t} F^{-1}$

1.2.a. $\quad \alpha=$ id

1.2.b. $n=2 m: \quad \alpha(X)=F X^{t} F^{-1}$

1.2.c. $\quad \alpha(X)=I_{p, q} X^{t} I_{u, v}$

1.3.a. $\quad \alpha=$ id

1.3.b. $\alpha(X)=X^{t}$

1.3.c. $\alpha(X)=I_{p, q} \bar{X}^{t} I_{u, v}$

2.1.a. $\quad \alpha(X)=I_{p, q} X I_{p, q}$

2.1.b. $n=2 m: \quad \alpha(X)=F X F^{-1}$

2.2.a. $\quad \alpha=$ id

2.2.b. $\quad \alpha(X)=I_{2 r, 2 s} X^{t} I_{2 r, 2 s}(r+s=m)$

3.1.a. $\quad \alpha(X)=F I_{p, q} X F I_{p, q}$

3.1.b. $\alpha(X)=\bar{X}=X^{t}$ 


$$
\begin{array}{ll}
\text { 3.2.a. } & \alpha=\mathrm{id} \\
\text { 3.2.b. } & \alpha(X)=F I_{p, q} X F I_{p, q} \\
\text { 4.1.a. } & \alpha(x+y)=I_{r, p-r} x+I_{s, q-s} y\left(x \in \mathbb{R}^{p}, y \in \mathbb{R}^{q}\right) \\
\text { 4.1.b. } & \text { even, } p=q: \quad \alpha=F \\
\text { 6.1.a. } \quad \alpha(X)=I_{k, l} X I_{i, j} \\
\text { 6.1.b. } p=2 r, q=2 s: \quad \alpha(X)=F_{r} X F_{s}^{-1} \\
\text { 6.2.a. } \quad \alpha(X)=I_{k, l} X I_{i, j} \\
\text { 6.2.b. } \alpha(X)=\bar{X} \\
\text { 7.1.a. } \quad \alpha(X)=I_{p, q} X I_{p, q}
\end{array}
$$

\subsection{Geometric part.}

4.2.0. Notation. The para-Hermitian symmetric space associated to a simple Jordan pair $V$ is denoted by $G / H=\operatorname{Co}(V) / \operatorname{Str}(V)$. The group $\operatorname{Str}(V)$ is is isomorphic to the structure group of $T$ and $\mathrm{Co}(V)$ to the conformal group of $T$. (This is explained in $[\mathrm{Be} 00]$.)

We denote by $X^{(\alpha)}=G^{(\alpha)} / H$ and $X^{(-\alpha)}=G^{(-\alpha)} / H$ the (c-dual) symmetric spaces associated to the modifications $T^{\alpha}$ and $T^{-\alpha}$ of a JTS $T$ as described in the algebraic classification. This agrees with the notation introduced in Be96b] and Be98 where we have defined these spaces by a construction using Jordan algebras. The determination of the conformal group and of the spaces $X^{( \pm \alpha)}$ from the data $T$ and $\alpha$ uses basically only linear algebra; we have given details for the classical matrix spaces in Be96b. A symmetric space of group type is denoted by $X=H \times H / H$ where a Lie group $H$ is identified with the diagonal in $H \times H$; its c-dual space is given by $H_{\mathbb{C}} / H$. The symbol $\mathbb{P}$ in front of a group means the quotient group w.r.t. scalars.

4.2.1. Spaces having invariant structures of all three types. These are the spaces $G / H=\operatorname{Co}\left(V_{\mathbb{C}}\right)_{o} / \operatorname{Str}\left(V_{\mathbb{C}}\right)_{o}$ where $V_{\mathbb{C}}$ is a complex Jordan pair. From Table 4.1.1 we get

$$
\begin{array}{lll}
V_{\mathbb{C}} & \multicolumn{1}{c}{G=\mathrm{Co}\left(V_{\mathbb{C}}\right)_{o}} & H=\operatorname{Str}\left(V_{\mathbb{C}}\right)_{o} \\
1 . & \mathbb{P} \mathrm{Gl}(2 n, \mathbb{C}) & \mathbb{P}(\mathrm{Gl}(n, \mathbb{C}) \times \mathrm{Gl}(n, \mathbb{C})) \\
2 . & \mathrm{Sp}(n, \mathbb{C}) & \mathrm{Gl}(n, \mathbb{C}) \\
3 . & \mathrm{SO}(4 m, \mathbb{C}) & \mathrm{Gl}(2 m, \mathbb{C}) \\
4 . & \mathrm{SO}(n+2, \mathbb{C}) & \mathrm{SO}(n, \mathbb{C}) \times \mathrm{SO}(2, \mathbb{C}) \\
5 . & E_{7} & E_{6} \times \mathbb{C}^{*} \\
6 . & \mathbb{P} \mathrm{Gl}(p+q, \mathbb{C}) & \mathbb{P}(\mathrm{Gl}(p, \mathbb{C}) \times \mathrm{Gl}(q, \mathbb{C})) \\
7 . & \mathrm{SO}(4 m+2, \mathbb{C}) & \mathrm{Gl}(2 m+1, \mathbb{C}) \\
8 . & E_{6} & \mathrm{SO}(10, \mathbb{C}) \times \mathbb{C}^{*}
\end{array}
$$

Any of the three complexification functors, applied to one of these spaces, yields a direct product of the space with itself. The space of type 4 admits a second Jordan extension, given by Table 4.2.4, 6.a. $(q=2)$.

4.2.2. Para-Hermitian symmetric spaces without invariant complex structure. These are the spaces $\operatorname{Co}(V)_{o} / \operatorname{Str}(V)_{o}$ with $V$ a Jordan pair from Table 4.1.2. 


$\begin{array}{lll}V \quad G=\mathrm{Co}(V)_{o} & H=\operatorname{Str}(V)_{o} \\ \text { 1.1. } \quad \mathrm{SU}(n, n) & \mathrm{Gl}(n, \mathbb{C}) / \mathbb{R}^{*} \\ \text { 1.2. } \quad \mathbb{P} \mathrm{Gl}(2 n, \mathbb{R}) & \mathbb{P}(\mathrm{Gl}(n, \mathbb{R}) \times \mathrm{Gl}(n, \mathbb{R})) \\ \text { 1.3. } n=2 m: \quad \mathbb{P} \mathrm{Gl}(2 m, \mathbb{H}) & \mathbb{P}(\mathrm{Gl}(m, \mathbb{H}) \times \mathrm{Gl}(m, \mathbb{H})) \\ \text { 2.1. } \quad \mathrm{Sp}(n, \mathbb{R}) & \mathrm{Gl}(n, \mathbb{R}) \\ \text { 2.2. } n=2 m: \quad \mathrm{Sp}(m, m) & \mathrm{Gl}(m, \mathbb{H}) \\ \text { 3.1. } \quad \mathrm{SO}^{*}(4 m) & \mathrm{Gl}(m, \mathbb{H}) \\ \text { 3.2. } n=2 m: \quad \mathrm{SO}(n, n) & \mathrm{Gl}(n, \mathbb{R}) \\ \text { 4.1. } \quad \mathrm{SO}(p+1, q+1) & \mathrm{SO}(p, q) \times \mathrm{SO}(1,1), p+q=n \\ \text { 5.1. } \quad E_{7(-25)} & E_{6(-25) \times \mathbb{R}^{+}} \\ \text {5.2. } \quad E_{7(7)} & E_{6(6)} \times \mathbb{R}^{+} \\ \text {6.1. } \quad \mathbb{P} \mathrm{Gl}(p+q, \mathbb{R}) \quad \mathbb{P}(\mathrm{Gl}(p, \mathbb{R}) \times \mathrm{Gl}(q, \mathbb{R})) \\ \text { 6.2. } p=2 r, q=2 s: \quad \mathbb{P} \mathrm{Gl}(r+s, \mathbb{H}) & \mathbb{P}(\mathrm{Gl}(r, \mathbb{H}) \times \mathrm{Gl}(s, \mathbb{H})) \\ \text { 7.1. } n=2 m+1: \quad \mathrm{SO}(n, n) & \mathrm{Gl}(n, \mathbb{R}) \\ \text { 8.1. } \quad E_{6(6)} & \mathrm{SO}(5,5) \times \mathbb{R}^{+} \\ \text {8.2. } \quad E_{6(-26)} & \mathrm{SO}(1,9) \times \mathbb{R}^{+}\end{array}$

The straight and the twisted complexification of the space labelled by $i . j$. is given by the space in Table 4.2.1. labelled by $i$. The space of type 4.1 has another Jordan extension given by Table 4.2.5, 6.1.a $(i=j=1)$. The spaces 1.1, 2.1, 3.1, 4.1 and 5.1 have invariant causal structures; they are known as causal symmetric spaces of Cayley type.

4.2.3. Pseudo-Hermitian symmetric spaces without invariant straight complex structure. These are the spaces $G^{(\alpha)} / H$ and $G^{(-\alpha)} / H$ belonging to the Hermitian JTS's $T^{\alpha}$ and $T^{-\alpha}$ from Table 4.1.3.

$\begin{array}{llll}\alpha & G^{(\alpha)} & G^{(-\alpha)} & H \\ \text { 1.A. } & \mathrm{Gl}(n, \mathbb{H}) & \mathrm{Gl}(2 n, \mathbb{R}) & \mathrm{Gl}(n, \mathbb{C}) \\ \text { 1.B. } & \mathrm{U}(p+v, q+u) & \mathrm{U}(p+u, q+v) & \mathrm{U}(u, v) \times \mathrm{U}(p, q) \\ \text { 2.A. } & \mathrm{Sp}(p, n-p) & \mathrm{Sp}(n, \mathbb{R}) & \mathrm{U}(p, n-p) \\ \text { 3.A. } & \mathrm{SO}(2 p, 4 m-2 p) & \mathrm{SO}^{*}(4 m) & \mathrm{U}(p, 2 m-p) \\ \text { 4.A. } & \mathrm{SO}(p-1, q+3) & \mathrm{SO}(p+1, q+1) & \mathrm{SO}(p-1, q+1) \times \mathrm{SO}(2) \\ \text { 4.B. } & \mathrm{SO}^{*}(2+2 q) & \mathrm{SO}^{*}(2+2 q) & \mathrm{SO}^{*}(2) \times \mathrm{SO}^{*}(2 q) \\ \text { 6.A. } & \mathrm{U}^{(l+i, k+j)} & \mathrm{U}(l+j, k+i) & \mathrm{U}(k, l) \times \mathrm{U}(i, j) \\ \text { 7.A. } & \mathrm{SO}^{*}(4 m-2) & \mathrm{SO}(2 p, 4 m+2-2 p) & \mathrm{U}(p, 2 m+1)\end{array}$

The straight complexification and twisted para-complexification of space $i . X$. is given by space $i$ in Table 4.2.1. The space of type 4.A carries a second Jordan extension, given by Table 4.2.5, 6.1.a $(i=2, j=0)$, and the space of type 4.B carries a second structure given by Table 4.2.5, 6.2.b $(p=1)$. The following are pseudo-Hermitian symmetric spaces of tube type in the sense of [FG96]: 1.A.+, 1.A.,- 1.B. $+(p=u, q=v), 2$.A.,+ 2. A. - , 3.A. + , 3.A.- - 4.A.+ .

4.2.4. Straight complex symmetric spaces with twist having no invariant twisted complex structure. They correspond to Table 4.1.4; here $X^{(\alpha)}$ is isomorphic to its c-dual $X^{(-\alpha)}$.

The twisted complexification and para-complexification of space $i . x$ is given by space $i$ in Table 4.2.1. The complex spheres carry three Jordan extensions: they appear in the above table as types 6.a $p=1$ and 4.a $q=0$, and also in Table 4.2.5, 


\begin{tabular}{|c|c|c|}
\hline$\alpha$ & $G^{(\alpha)} \cong G^{(-\alpha)}$ & $H$ \\
\hline 1.a. & $H \times H$ & $H=\operatorname{Gl}(n, \mathbb{C})$ \\
\hline 1.b. & $\mathrm{SO}(2 n, \mathbb{C})$ & $\mathrm{SO}(n, \mathbb{C}) \times \mathrm{SO}(n, \mathbb{C})$ \\
\hline 1.c. & $\operatorname{Sp}(2 m, \mathbb{C})$ & $\operatorname{Sp}(m, \mathbb{C}) \times \operatorname{Sp}(m, \mathbb{C})$ \\
\hline 2.a. & $\operatorname{Gl}(n, \mathbb{C})$ & $\mathrm{SO}(n, \mathbb{C})$ \\
\hline 2.b. & $H \times H$ & $H=\operatorname{Sp}(m, \mathbb{C})$ \\
\hline 3.a. & $\operatorname{Gl}(2 m, \mathbb{C})$ & $\operatorname{Sp}(m, \mathbb{C})$ \\
\hline 3.b. & $H \times H$ & $H=\mathrm{SO}(2 m, \mathbb{C})$ \\
\hline & $\mathrm{SO}(p+1, \mathbb{C}) \times \mathrm{SO}(q+1, \mathbb{C})$ & $\mathrm{SO}(p, \mathbb{C}) \times \mathrm{SO}(q, \mathbb{C})$ \\
\hline & $\mathrm{SO}(p+q, \mathbb{C})$ & $\mathrm{SO}(p, \mathbb{C}) \times \mathrm{SO}(q, \mathbb{C})$ \\
\hline 6.b. & $p=2 r, q=2 s: \operatorname{Sp}(r+s, \mathbb{C})$ & $\operatorname{Sp}(r, \mathbb{C}) \times \operatorname{Sp}(s, \mathbb{C})$ \\
\hline & $H \times H$ & $H=\mathrm{SO}(2 m+1, \mathbb{C})$ \\
\hline
\end{tabular}

4.1.b. Further, the spaces of type 4.a carry also structures of reducible Jordan extensions, namely those given by direct product of the structures on the complex spheres. Finally, the space of type 6.a $q=2$ carries a second Jordan extension given by Table 4.2.1, 4 .

4.2.5. Symmetric spaces with twist having no invariant complex or paracomplex structure. These are the spaces $X^{(\alpha)}$ and $X^{(-\alpha)}$ corresponding to Table 4.1.5. In the last column we list the twisted complexification of the space: it is given by the line of Table 4.2.3 with the indicated label. The additional parameter "-" means that in the corresponding line of Table 4.2.3, $\alpha$ and $-\alpha$ have to be exchanged. For example, the twisted complexification of the $\operatorname{group} \operatorname{Sp}(m, \mathbb{R})$ (line 2.1.b, first and third column) can be found in Table 4.2.3, line 2.A, second and third column with the indicated parameters: it is the $\operatorname{space} \operatorname{Sp}(2 m, \mathbb{R}) / \mathrm{U}(m, m)$. For some symplectic series one has to take twice the parameters as given in Table 4.2.3 in order to get the twisted complexification; this is indicated by "double param."

The twisted para-complexification of space $i . j . x$ is given by space $i . j$ of Table 4.2.2, and the "double complexification" is given by space $i$ of Table 4.2.1.

\begin{tabular}{|c|c|c|c|}
\hline$\alpha \quad G^{(\alpha)}$ & $G^{(-\alpha)}$ & $H$ & $h \mathbb{C}$ \\
\hline 1.1.a. $H \times H$ & $H_{\mathbb{C}}=\operatorname{Gl}(n, \mathbb{C})$ & $H=\mathrm{U}(p, q)$ & 1.B, $v=p, u=q$ \\
\hline 1.1.b. $\mathrm{SO}^{*}(2 n)$ & $\mathrm{SO}(n, n)$ & $\mathrm{SO}(n, \mathbb{C})$ & 1.A \\
\hline 1.1.c. $\operatorname{Sp}(2 m, \mathbb{R})$ & $\operatorname{Sp}(m, m)$ & $\operatorname{Sp}(m, \mathbb{C})$ & 1.A.- \\
\hline 1.2.a. $H_{\mathbb{C}}=\operatorname{Gl}(n, \mathbb{C})$ & $H \times H$ & $H=\operatorname{Gl}(n, \mathbb{R})$ & 1.A \\
\hline 1.2.b. $\operatorname{Sp}(2 m, \mathbb{R})$ & $\operatorname{Sp}(2 m, \mathbb{R})$ & $\operatorname{Sp}(m, \mathbb{R}) \times \operatorname{Sp}(m, \mathbb{R})$ & 1.B.-,$p=q=u=v=m$ \\
\hline 1.2.c. $\mathrm{SO}(p+v, q+u)$ & $\mathrm{SO}(p+u, q+v)$ & $\mathrm{SO}(u, v) \times \mathrm{SO}(p, q)$ & 1.B \\
\hline 1.3.a. $H_{\mathbb{C}}=\operatorname{Gl}(2 m, \mathbb{C})$ & $H \times H$ & $H=\mathrm{Gl}(m, \mathbb{H})$ & 1.A.- \\
\hline 1.3.b. $\mathrm{SO}^{*}(4 m)$ & $\mathrm{SO}^{*}(4 m)$ & $\mathrm{SO}^{*}(2 m) \times \mathrm{SO}^{*}(2 m)$ & 1.B.-,$p=q=u=v=m$ \\
\hline 1.3.c. $\operatorname{Sp}(p+v, q+u)$ & $\operatorname{Sp}(p+u, q+v)$ & $\operatorname{Sp}(u, v) \times \operatorname{Sp}(p, q)$ & 1.B, double param. \\
\hline 2.1.a. $\mathrm{U}(p, q)$ & $\operatorname{Gl}(n, \mathbb{R})$ & $\mathrm{O}(p, q)$ & 2.A \\
\hline 2.1.b. $H \times H$ & $H_{\mathbb{C}}=\operatorname{Sp}(m, \mathbb{C})$ & $H=\operatorname{Sp}(m, \mathbb{R})$ & 2.A.-,$p=m=n / 2$ \\
\hline 2.2.a. $\mathrm{U}(m, m)$ & $\operatorname{Gl}(m, \mathbb{H})$ & $\mathrm{SO}^{*}(2 m)$ & 2.A.-,$p=m=n / 2$ \\
\hline 2.2.b. $H \times H$ & $H_{\mathbb{C}}=\operatorname{Sp}(m, \mathbb{C})$ & $H=\operatorname{Sp}(r, m-r)$ & 2.A, $p=2 r, n=2 m$ \\
\hline 3.1.a. $\mathrm{U}(2 p, 2 q)$ & $\operatorname{Gl}(m, \mathbb{H})$ & $\operatorname{Sp}(p, q)$ & 3.A \\
\hline 3.1.b. $H \times H$ & $H_{\mathbb{C}}=\mathrm{SO}(2 m, \mathbb{C})$ & $H=\mathrm{SO}^{*}(2 m)$ & 3.A.-, $p=m$ \\
\hline 3.2.a. $\mathrm{U}(m, m)$ & $\operatorname{Gl}(2 m, \mathbb{R})$ & $\operatorname{Sp}(m, \mathbb{R})$ & 3.A.-, $p=m$ \\
\hline 3.2.b. $H \times H$ & $H_{\mathbb{C}}=\operatorname{SO}(2 m, \mathbb{C})$ & $H=\mathrm{SO}(p, 2 m-p)$ & 3.A \\
\hline 4.1.b. $\mathrm{SO}(p+1, \mathbb{C})$ & $\mathrm{SO}(p+1, \mathbb{C})$ & $\mathrm{SO}(p, \mathbb{C})$ & 4. B, $p=q$ \\
\hline 6.1.a. $\operatorname{SO}(l+i, k+j)$ & $\mathrm{SO}(l+j, k+i)$ & $\mathrm{SO}(k, l) \times \mathrm{SO}(i, j)$ & $6 . \mathrm{A}$ \\
\hline 6.1.b. $\operatorname{Sp}(r+s, \mathbb{R})$ & $\operatorname{Sp}(r+s, \mathbb{R})$ & $\operatorname{Sp}(r, \mathbb{R}) \times \operatorname{Sp}(s, \mathbb{R})$ & 6.A, $k=l=r, i=j=s$ \\
\hline 6.2.a. $\operatorname{Sp}(l+i, k+j)$ & $\operatorname{Sp}(l+j, k+i)$ & $\operatorname{Sp}(k, l) \times \operatorname{Sp}(i, j)$ & 6.A, double param. \\
\hline 6.2.b. $\mathrm{SO}^{*}(2 p+2 q)$ & $\mathrm{SO}^{*}(2 p+2 q)$ & $\mathrm{SO}^{*}(2 p) \times \mathrm{SO}^{*}(2 q)$ & 6.A, $k=l=2 p, i=j=2 q$ \\
\hline 7.1.a. $H \times H$ & $H_{\mathbb{C}}=\mathrm{SO}(2 m+1, \mathbb{C})$ & $H=\mathrm{SO}(p, 2 m+1-p)$ & 7.A.- \\
\hline
\end{tabular}


Finally, in case 4.1.a we have

$$
\begin{aligned}
G^{(\alpha)} & =\mathrm{SO}(r-1, s+1) \times \mathrm{SO}(q-r+2, p-s), G^{(-\alpha)} \\
& =\mathrm{SO}(r-1, s) \times \mathrm{SO}(q-r+1, p-s),
\end{aligned}
$$

$H=\mathrm{SO}(r, s) \times \mathrm{SO}(q-r-1, p-s+1)$, and the twisted complexification is given by 4 .A.

Remarks. 1. The spaces with numbers $1.1,2.1,3.1$ and $4.1(p=1)$ are causal symmetric spaces with a causal structure given by a symmetric cone (cf. [Be96b]).

2. The following spaces have several Jordan extensions: 6.2.b (cf. Table 4.2.3), 6.1.a for $i=2, j=0$ (cf. Table 4.2.3) and for $i=j=1$ (cf. Table 4.2.2), 4.1.b (cf. Table 4.2.4), and 4.1.a for $r=1, s=0$ which appears also in 6.1.a with $i=1, j=0$. Among the latter are the real hyperbolic spaces. Finally, the spaces of 4.1.a also have structures of reducible Jordan extensions. The reader may have noticed that all multiple structures come out of the relation between types 4 ("classical" conformal space) and $6, p=1$ (projective space).

3. The trivial one-dimensional LTS has a continious family of Jordan extensions, all scalar multiples of $T(x, y, z)=x y z$. The corresponding complexifications of the real line can be understood as the hyperbolic plane which is deformed into the flat complex plane as the positive scalar factor tends to zero, and similarly with the Riemann sphere if the scalar factor is negative. The corresponding paracomplexfication is the one-sheeted hyperboloid.

4.2.6. A semi-exceptional space. It can be deduced from [Ma73, p. 416], that the symmetric spaces of classical type $M=\mathrm{Sl}(4, \mathbb{H}) / \mathrm{Sp}(3,1)$ and $M_{\mathbb{C}}=\mathrm{Sl}(8, \mathbb{C}) /$ $\operatorname{Sp}(4, \mathbb{C})$ have exceptional Hermitian complexifications, namely

$$
M_{h \mathbb{C}}=E_{7(2)} /\left(E_{6(2)} \times S^{1}\right) \quad \text { and } \quad M_{p h \mathbb{C}}=\operatorname{Co}(\operatorname{Herm}(3, \mathbb{O})) / \operatorname{Str}(\operatorname{Herm}(3, \mathbb{O}))
$$

(cf. Table 4.2.2). Remark that central extensions of $M$ and $M_{\mathbb{C}}$ have classical Hermitian complexifications (cf. Table 4.2.5, 3.1.a and 4.2.4, 3.a) corresponding to the equation of dimensions $\operatorname{dim} \operatorname{Herm}(3, \mathbb{O})=\operatorname{dim} \operatorname{Herm}(4, \mathbb{H})-1$. The fact that classical LTS's may have exceptional Jordan extensions has also been observed by Loos [Lo77, 11.19] and Neher [N85].

\section{REFERENCES}

[B57] M. Berger, Les espaces symétriques non compacts, Ann. Sci. Ec. Norm. Sup. 74 (3) (1957), 85-177. MR 21:3516

[Be96a] W. Bertram, Un théorème de Liouville pour les algèbres de Jordan, Bull. Soc. Math. France 124 (1996), 299-327. MR 97g:17030

[Be96b] W. Bertram, On some causal and conformal groups, J. Lie Theory 6 (1996), 215-244. MR 98d:53076

[Be98] W. Bertram, W, Algebraic Structures of Makarevič Spaces. I, Transformation Groups 3, vol.1 (1998), 3-32. MR 99c:32047

[Be99] W. Bertram, The geometry of Jordan- and Lie structures, Habilitationsschrift (Clausthal 1999).

[Be00] W. Bertram, Conformal group and fundamental theorem for a class of symmetric spaces, Math. Z. 233 (2000), 39-73. CMP 2000:07

[FG96] J. Faraut and S. Gindikin, Pseudo-Hermitian symmetric spaces of tube type, Progress in Nonlinear Diff. Equat. 20 (1996), 123-154. MR 97i:32045

[Jac51] N. Jacobson, General representation theory of Jordan algebras, Trans. Amer. Math. Soc. 70 (1951), 509-530. MR 12:797d

[Hel62] S. Helgason, Differential Geometry and Symmetric Spaces, Academic Press (1962). MR 26:2986 
[KanKo85] S. Kaneyuki and M.Kozai, Paracomplex structures and affine symmetric spaces, Tokyo J. Math 8 (1985), 81-98. MR 87c:53078

[Koh65] S. Koh, On affine symmetric spaces, Trans. Amer. Math. Soc. 119 (1965), 291-309. MR 32:1643

[KoNo69] S. Kobayashi and K. Nomizu, Foundations of Differential Geometry, vol. II, Interscience (New York 1969). MR 38:6501

[Lo69] O. Loos, Symmetric Spaces I, Benjamin (New York 1969). MR 39:3659

[Lo75] O. Loos, Jordan pairs, Springer Lecture Notes in Mathematics, vol. 460 (New York 1975). MR 56:3071

[Lo77] O. Loos, Bounded symmetric domains and Jordan pairs, Lecture Notes (Irvine 1977).

[Lo85] O. Loos, Charakterisierung symmetrischer R-Räume durch ihre Einheitsgitter, Math. Z. 189 (1985), 211-226. MR 86j:53077

[Ma73] B.O. Makarevič, Open symmetric orbits of reductive groups in symmetric $R$-spaces, Math. USSR Sbornik 20 (1973), 406-418.

[N80] E. Neher, Klassifikation der einfachen reellen speziellen Jordan-Tripelsysteme, Manuscripta Math. 31 (1980), 197-215. MR 82a:17011

[N81] E. Neher, E, Klassifikation der einfachen reellen Ausnahme-Jordan-Tripelsysteme, J. reine angew. Math. 322 (1981), 145-169. MR 82g:17017

[N85] E. Neher, On the classification of Lie and Jordan triple systems, Comm. Algebra 13 (1985), 2615-2667. MR 87c:17036

[Sa80] I. Satake, Algebraic structures of symmetric domains, Iwanami Shoten (Princeton 1980). MR 82i:32003

Institut Elie Cartan, Département de Mathématiques, Université Henri Poincaré (Nancy I), B.P. 239, 54506 Vandeeuvre-les-Nancy Cedex, France

E-mail address: bertram@iecn.u-nancy.fr 